\title{
Advanced Monte Carlo methods for barrier and related exotic options
}

\author{
Emmanuel Gobet ${ }^{a}$ \\ ${ }^{a}$ Laboratoire Jean Kuntzmann, Université de Grenoble and CNRS, BP 53, 38041 \\ Grenoble cedex 9, FRANCE
}

First version: 2nd January 2008

This version 31st July 2008

\begin{abstract}
In this work, we present advanced Monte Carlo techniques applied to the pricing of barrier options and other related exotic contracts. It covers in particular the Brownian bridge approaches, the barrier shifting techniques (BAST) and their extensions as well. We leverage the link between discrete and continuous monitoring to design efficient schemes, which can be applied to the Black-Scholes model but also to stochastic volatility or Merton's jump models. This is supported by theoretical results and numerical experiments.
\end{abstract}

\section{Introduction}

In this paper, we review and extend advanced techniques for the valuation of barrier options (initially introduced by Merton [22]) and other financial contracts, whose activation depends on whether an underlying asset has reached or not a specified level (the barrier). From the Monte Carlo point of view, these types of payoff are difficult to simulate because they are strongly path dependent and they are discontinuous with respect to the path of the monitored process. The option prototype is the Down and Out Call (DOC), which is a European Call with strike $K$ and expiration date $T$, but it is paid if and only if the asset $X$ has not reached the lower level $D$ before expiration. This

Email address: emmanuel.gobet@imag.fr (Emmanuel Gobet). 
is a knock out option, whose payoff can be written as follows:

$$
\Psi_{T}=\mathbf{1}_{\forall t<T: X_{t}>D}\left(X_{T}-K\right)_{+}
$$

On the other hand, a knock in option comes into effect only when the barrier is reached. However, a knock out option plus a knock in option with same parameters readily gives a vanilla option; thus, by absence of arbitrage, one needs to focus on only one type and this will the knock out in what follows. We mention that all these options are also known as trigger options. Additionally, a rebate can be paid at the trigger when the option ceases to exist (in the case of a knock out option).

The type of barrier options can be widened with many respects. First, it can be designed for several assets simultaneously, with barriers on each asset. Double barriers (lower and upper) or time dependent barriers are complementary choices. Also, there may be a discrete time monitoring instead of a continuous one: for instance, the option is not knocked out if the asset remains above the barrier, daily at a fixing hour. Moreover as shown hereafter, it is quite fruitful to connect the discrete and continuous monitoring.

At this level of description, the knowledge of the stochastic model does not matter. It could be given by the solution of a Brownian stochastic differential equations (SDE in short), and it could include jumps as well. It could be related to Forex markets, where these options are of the most popular types, but related assets can be traded on Equity or Fixed Income markets as well. Usually they are over-the-counter contracts. Within the structured products, it is also quite common to meet barrier type options.

The problem to simulate such payoff $\Psi_{T}$ is that one has to compare the value of the asset and of the barrier at any time before expiration date $T$, to decide whether the option is knocked out or not, whereas one is able to simulate the path only at a fixed number $N$ of times (the monitoring dates) $\left(t_{i}\right)_{1 \leq i \leq N}$. If the monitoring frequency is high, one expects that the payoffs $\Psi_{T}$ and $\Psi_{T}^{N}=\mathbf{1}_{\forall t_{i}<T: X_{t_{i}}>D}\left(X_{T}-K\right)_{+}$are close from each other. This may be false for some simulation scenarii but true when one takes the expectation, which is the criterion of interest for valuation. However, the monitoring bias $\mathbb{E} \Psi_{T}^{N}-\mathbb{E} \Psi_{T}$ shrinks to 0 quite slowly (as $N^{-1 / 2}$, see the references hereafter) and besides this, it is positive because we may not detect that a trigger has occurred between two successive dates. The bad consequence is to systematically overestimate the price (here, we omit the discount factor when referring to the price). 
To overcome this difficulty, there are two numerical strategies.

- Either to discretize the state space of the asset $X$ (or its logarithm). Then, simulate the successive hitting times of this space grid is a smart way (see Rogers and Stapleton [25]) to well control the behavior of the path, and thus to reduce the simulation bias. Actually to be optimal, one should choose carefully the grid so that the barrier lies in the grid. This is closely related to binomial or multinomial tree methods. We refer to Boyle and Lau [4], Boyle and Tian [5], Cheuk and Vorst [9], Derman et al. [11], Ritchken [24], Rogers and Stapleton [25], Rubinstein and Reiner [26]. This approach becomes difficult to design, especially for several dependant assets and when jumps may occur.

- Or to take into account that the option may be knocked out between monitoring times. One possibility is to simulate the trigger event conditionally on the known values of $X$. This is known as Brownian bridge techniques and this dates back to Baldi [2]. This and related refinements will be discussed in Section 1. Another possibility consists in shifting the trigger in order to compensate the overestimation. This idea, initially put in a financial framework by Broadie et al. [6] for a single asset in the Black-Scholes model, can be extended in many ways (multi assets, local volatility, time dependant barrier, jumps...). Actually it turns out to be quite flexible. It is discussed in Section 2.

Section 3 brings together several financial examples, where we illustrate how the previous methods perform. We do not present results related to the computations of Greeks: see for instance Gobet [15] and the references therein.

\section{Brownian bridge techniques}

\subsection{A toy example}

Before coming into advanced techniques designed for sophisticated models, we begin with the simplest financial example of Black-Scholes model with constant coefficients, used to price a Down and Out Call option. Thus, under the risk-neutral probability the dynamics of the underlying asset $X$ is given by

$$
\frac{\mathrm{d} X_{t}}{X_{t}}=\mu \mathrm{d} t+\sigma \mathrm{d} W_{t}
$$


where $W$ is a standard Wiener process. Here $\sigma$ is the volatility, $\mu$ is the drift under the risk-neutral probability (generally equal to the interest rate minus the continuous dividend rate if $X$ is an equity, or to the difference of interest rates of both economies if $X$ is an exchange rate). To simplify even more, consider that the discount factor equals 1 (zero interest rate). Then, the fair price is given by the expectation

$$
\mathbb{E}\left(\mathbf{1}_{\forall t<T: X_{t}>D}\left(X_{T}-K\right)_{+}\right),
$$

that we may evaluate by Monte Carlo methods. Of course, in this toy example, one has a closed formula for this price (see Rubinstein and Reiner [26] and Equation (27) below), which is useful for numerics to check the validity of a procedure.

Without the simulation of the trigger. For the simulation, we may proceed as follows.

- firstly, simulate $X_{T}$ by ${ }^{1}$

$$
X_{T} \stackrel{d}{=} x_{0} \exp \left(\left(\mu-\frac{1}{2} \sigma^{2}\right) T+\sigma \sqrt{T} Z\right)
$$

where $Z$ is Gaussian variable with zero mean and unit variance.

- secondly, compute (analytically) the conditional trigger probability

$$
p(x, y, T, D,|\sigma|)=\mathbb{P}\left(\exists t<T: X_{t} \leq D \mid X_{0}=x, X_{T}=y\right)
$$

and as an output, take

$$
\left[1-p\left(x_{0}, X_{T}, T, D,|\sigma|\right)\right]\left(X_{T}-K\right)_{+} .
$$

Draw many independent simulations of the output, and by averaging them out, one gets an approximation of

$$
\begin{aligned}
& \mathbb{E}\left(\left[1-p\left(x_{0}, X_{T}, T, D,|\sigma|\right)\right]\left(X_{T}-K\right)_{+}\right) \\
& =\mathbb{E}\left(\mathbb{E}\left(\mathbf{1}_{\forall t<T: X_{t}>D} \mid X_{0}, X_{T}\right)\left(X_{T}-K\right)_{+}\right) \\
& =\mathbb{E}\left(\mathbf{1}_{\forall t<T: X_{t}>D}\left(X_{T}-K\right)_{+}\right) .
\end{aligned}
$$

This leads to an unbiased Monte Carlo procedure, provided that we are able to compute the conditional trigger probability. Actually, this quantity is explicit

$\overline{1 U \stackrel{d}{=}} V$ means that $U$ and $V$ have the same distribution. 
and it is given by

$$
p(x, y, T, D,|\sigma|)= \begin{cases}1 & \text { if } x \text { or } y \text { are below } D \\ \exp \left(-2 \frac{\log (x / D) \log (y / D)}{|\sigma|^{2} T}\right) & \text { otherwise }\end{cases}
$$

This expression easily follows from the known distribution of the value of a Brownian motion and of its running maximum at a given time (see Revuz and Yor [23]). Actually, this is equal to the probability that a Brownian bridge (namely $\left(\log \left(X_{t}\right)\right)_{t}$ conditionally to $\log \left(X_{0}\right)$ and $\log \left(X_{T}\right)$ ) reaches the level $\log (D)$ : this gives the label of such approach as Brownian bridge techniques. It is worth noticing that the conditional trigger probability does not depend on the drift $\mu$.

\section{PSEUDO-CODE}

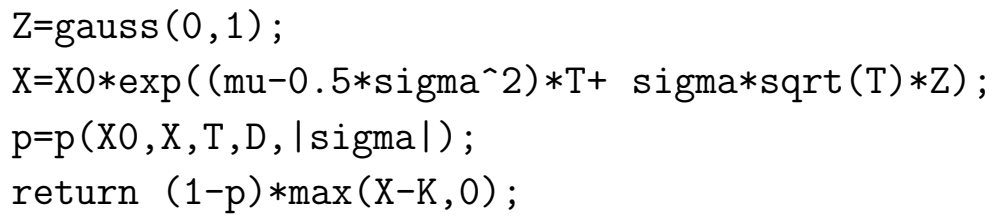

With the simulation of the trigger. In this toy example, to generate a scenario we only need to draw one random variable (i.e. $Z$ ) and to weight the call payoff by the non trigger probability. Note that one could also simulate the trigger by taking as an output

$$
\mathbf{1}_{U>p\left(0, x_{0}, X_{T}, T, D,|\sigma|\right)}\left(X_{T}-K\right)_{+}
$$

with an extra random variable $U$, independent of $Z$ and uniformly distributed on $[0,1]$. The procedure is still unbiased since the expectation remains unchanged. Only the variance is modified and it is now larger because the variance of the conditional expectation is smaller than the variance. Thus, the confidence interval is wider and on this example, simulating the trigger is not relevant for the computational efficiency.

\subsection{Easy extensions}

From the simple principle described above, we can derive several easy extensions, which allows us to handle less specific payoffs. Whereas in the previous examples there was no advantage to generate the underlying asset along a time grid, it is no longer true if the coefficients $\mu, \sigma$ are time-dependant, say 
piece-wise constant on each sub-intervals ${ }^{2}\left[t_{i}, t_{i+1}\left[\right.\right.$ and equal to $\mu_{i}$ and $\sigma_{i}$. In that case, one has firstly to generate $\left(X_{t_{i}}\right)_{i}$ at those times

$$
X_{t_{i+1}} \stackrel{d}{=} X_{t_{i}} \exp \left(\left(\mu_{i}-\frac{1}{2} \sigma_{i}^{2}\right) \Delta_{i}+\sigma_{i} \sqrt{\Delta_{i}} Z_{i}\right)
$$

with i.i.d. random variables $Z_{i} \stackrel{d}{=} Z$. Then, as an output, take

$$
\left(X_{T}-K\right)_{+} \prod_{i=0}^{N-1}\left(1-p\left(X_{t_{i}}, X_{t_{i+1}}, \Delta_{i}, D,\left|\sigma_{i}\right|\right)\right)
$$

where the function $p$ is still defined by (2).

\section{PSEUdO-CODE}

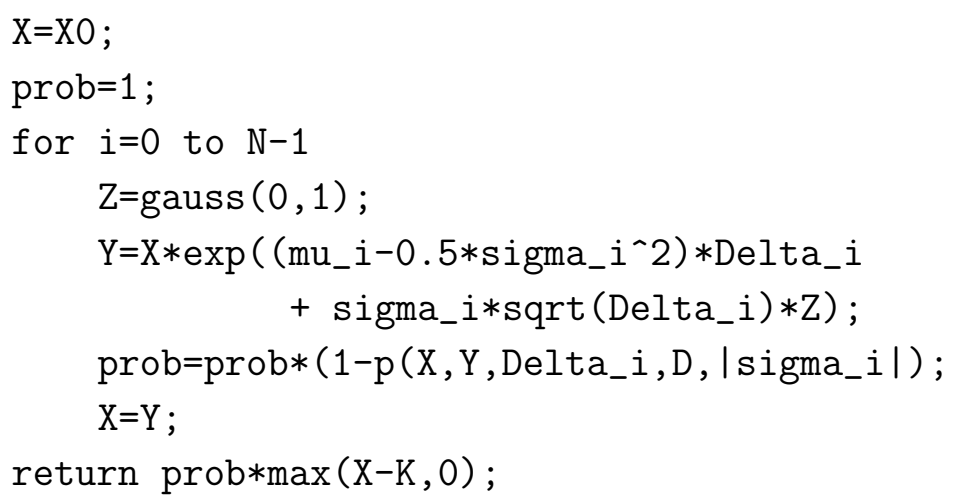

If the barrier $D$ is time dependant and piecewise constant as well, at each time step one has to evaluate $p\left(X_{t_{i}}, X_{t_{i+1}}, \Delta_{i}, D_{i},\left|\sigma_{i}\right|\right)$ instead of $p\left(X_{t_{i}}, X_{t_{i+1}}, \Delta_{i}, D,\left|\sigma_{i}\right|\right)$. Then, the procedure remains unbiased.

If there is an upper barrier $U$, we proceed very analogously, except that the conditional trigger probability $p$ is now given by

$$
p(x, y, \Delta, U,|\sigma|)= \begin{cases}1 & \text { if } x \text { or } y \text { are above } U \\ \exp \left(-2 \frac{\log (x / U) \log (y / U)}{|\sigma|^{2} \Delta}\right) & \text { otherwise }\end{cases}
$$

As in the toy example, we could alternatively simulate the trigger event. In the same way it slightly increases the variance of simulations. But now, the pseudo-code may be computationally cheaper since the loop over $i$ can be stopped as soon as the option is knocked out.

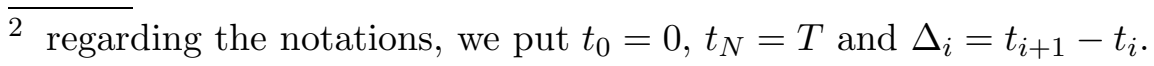


For a double barrier option (with down and up barriers $D$ and $U$ ), the expression for $p$ is still explicit:

$$
p(x, y, \Delta, D, U,|\sigma|)=\left\{\begin{array}{l}
1 \quad \text { if } x \text { or } y \text { are above } U \text { or if } x \text { or } y \text { are below } D \\
\sum_{k=-\infty}^{+\infty}\left[\exp \left(-2 \frac{k \log (U / D)(k \log (U / D)+\log (y / x))}{|\sigma|^{2} \Delta}\right)\right. \\
\left.-\exp \left(-2 \frac{(k \log (U / D)+\log (x / U))(k \log (U / D)+\log (y / U))}{|\sigma|^{2} \Delta}\right)\right] \text { otherwise. }
\end{array}\right.
$$

However, from large deviation arguments (see Baldi [2]) we know that one has to consider only the barrier which is the closest from $x$ and $y$ (and neglect the other one) and at a distance of order $|\sigma| \sqrt{\Delta_{i}}$; then, the evaluation of $p$ as a series boils down to the computation of one term. For a dense time grid $\left(\Delta_{i}\right.$ small), there is no possible ambiguity for such choice of the closest barrier but it may be questionable if $\Delta_{i}$ is not small ( $X_{t_{i}}$ may be close to $U$ and $X_{t_{i+1}}$ close to $D)$.

Notice that it is also straightforward to take into account in the simulation procedure that a jump component lies in the dynamics of $X$. For instance, if

$$
\frac{\mathrm{d} X_{t}}{X_{t^{-}}}=\mu \mathrm{d} t+\sigma \mathrm{d} W_{t}+Y_{t} \mathrm{~d} N_{t}
$$

where $N$ is a Poisson process with parameter $\lambda$ and $Y$ stands for the random jumps, we proceed as follows. Simulate the $k=N_{T}$ jump times $\left(\tau_{i}\right)_{i}$ up to time $T\left(\tau_{0}=0\right)$. Between two jumps, $X$ behaves like a geometric Brownian motion for which we can apply Brownian bridge techniques. This heuristic is fully justified by the fact that the Brownian part and the jump part are independent. Thus, we average out independent simulations of

$$
\begin{aligned}
\left(X_{T}-K\right)_{+} & \left(1-p\left(X_{\tau_{k}}, X_{T}, T-\tau_{k}, D, U,|\sigma|\right)\right) \\
& \times \prod_{i=0}^{k-1}\left(1-p\left(X_{\tau_{i}}, X_{\tau_{i+1}^{-}}, \tau_{i+1}-\tau_{i}, D, U,|\sigma|\right)\right)
\end{aligned}
$$

to get asymptotically and without bias the required expectation.

\subsection{Further approximations}

Up to now, we have described only unbiased procedures. But too often, models and payoffs are so complex that approximations are necessary to get through the numerics. 
Firstly, when the underlying process has a fairly general dynamics such as

$$
\mathrm{d} X_{t}=\mu\left(t, X_{t}\right) \mathrm{d} t+\sigma\left(t, X_{t}\right) \mathrm{d} W_{t}
$$

(with matrix notations) with non constant (or non linear) coefficients $\mu$ and $\sigma$, it is likely that one can not simulate exactly the process at given times. One alternative is to use the Euler scheme (based on a regular grid $\left(t_{i}\right)_{0 \leq i \leq N}$ with time step $\Delta=T / N)$, which is defined by $X_{0}^{N}=X_{0}$ and

$$
X_{t_{i+1}}^{N}=X_{t_{i}}^{N}+\mu\left(t_{i}, X_{t_{i}}^{N}\right) \Delta+\sigma\left(t_{i}, X_{t_{i}}^{N}\right)\left(W_{t_{i+1}}-W_{t_{i}}\right)
$$

If $X$ is a coordinate-wise positive process, it is better to use an Euler scheme on the $\log$-process $\log (X)$ to keep this positivity property. Regarding the Brownian bridge techniques, they are still applicable because on each interval $\left[t_{i}, t_{i+1}\left[, X^{N}\right.\right.$ is a Brownian motion with drift: $X_{t}^{N}=X_{t_{i}}^{N}+\mu\left(t_{i}, X_{t_{i}}^{N}\right)(t-$ $\left.t_{i}\right)+\sigma\left(t_{i}, X_{t_{i}}^{N}\right)\left(W_{t}-W_{t_{i}}\right)$. Thus, $\left(X_{t}^{N}\right)_{t_{i} \leq t \leq t_{i+1}}$ conditionally to $X_{t_{i}}^{N}=x$ and $X_{t_{i+1}}^{N}=y$ has the same law as $\left(\bar{x}_{t}=x+\sigma\left(t_{i}, x\right)\left(W_{t}-W_{t_{i}}\right)\right)_{t_{i} \leq t \leq t_{i+1}}$ conditionally to $\bar{x}_{t_{i+1}}=y$ (remind that the law of the Brownian bridge does not depend anymore of the initial drift). Now suppose for instance that the trigger is made via a knock out on the first asset $X_{1}$ at the lower level $D$, and that the payoff has the general form $f\left(X_{T}\right)$. Thus, the conditional trigger probability of the Euler scheme is given by

$$
\mathbb{P}\left(\exists t \in\left[t_{i}, t_{i+1}\right]: X_{1, t}^{N} \leq D \mid X_{t_{i}}^{N}, X_{t_{i+1}}^{N}\right)=p\left(X_{t_{i}}^{N}, X_{t_{i+1}}^{N}, \Delta, D,\left|\sigma_{1}\left(t_{i}, X_{t_{i}}^{N}\right)\right|\right)
$$

with $\sigma_{1}(\cdot)$ is the diffusion coefficient of $X_{1}$ (equal to the first row of $\sigma(\cdot)$ ) and

$$
p(x, y, \Delta, D, \bar{\sigma})= \begin{cases}1 & \text { if } x_{1} \text { or } y_{1} \text { are below } D \\ \exp \left(-2 \frac{\left(x_{1}-D\right)\left(y_{1}-D\right)}{\bar{\sigma}^{2} \Delta}\right) & \text { otherwise }\end{cases}
$$

Then, as an output take

$$
f\left(X_{T}^{N}\right) \prod_{i=0}^{N-1}\left(1-p\left(X_{t_{i}}^{N}, X_{t_{i+1}}^{N}, \Delta, D,\left|\sigma_{1}\left(t_{i}, X_{t_{i}}^{N}\right)\right|\right)\right)
$$

analogously to (3). 


\section{PSEUdo-CODE}

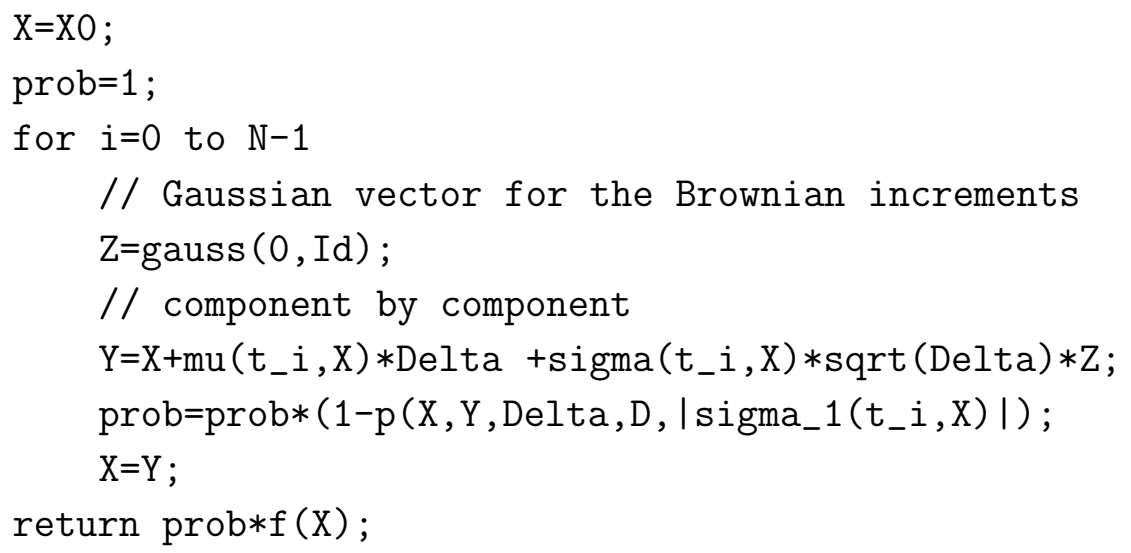

In this situation, the Monte Carlo procedure is biased because we use the Euler scheme. Indeed $\left(X_{t_{i}}^{N}\right)_{0 \leq i \leq N}$ are only approximative simulation values of $\left(X_{t_{i}}\right)_{0 \leq i \leq N}$ : the accuracy in $L_{p}$ norm is of order $\Delta^{1 / 2}$ (i.e. $\| \sup _{i \leq N} \mid X_{t_{i}}^{N}-$ $\left.X_{t_{i}} \mid \|_{p}=O\left(\Delta^{1 / 2}\right)\right)$. But one knows that for the evaluation of $\mathbb{E}\left(f\left(X_{T}\right)\right)$, the accuracy becomes a $O(\Delta)$ (this is the weak error, see Bally and Talay [3] for the mathematical analysis). The extension of this error analysis to barrier options has been carried out in Gobet [13] and it is shown that the weak error is still of order $\Delta$. Hence, provided that one suitably weights the payoff $f$ by the conditional trigger probability of the Euler scheme, the simulation bias is as small as if there was no barrier. It also means that it is not worth using a Milshtein scheme because the weak error has the same magnitude (in addition, we recall that the Milshtein scheme may be harder to use in general models because of restrictive conditions on $\sigma$ and its derivatives).

The crucial point in the above arguments is that one can analytically compute the trigger probability of the Euler scheme bridge (or equivalently of a Brownian bridge). For upper barrier and double barriers, this is possible as explained in paragraph 1.2. Difficulties really arise when barriers are multiple. When the trigger is associated to the first exit time of a given domain $\mathcal{D}$ (i.e. the option payoff is of the form $\mathbf{1}_{\forall t<T: X_{t} \in \mathcal{D}} f\left(X_{T}\right)$ ), one may use large deviations arguments (see Baldi [2]) to get an accurate approximation of

$$
p\left(x, y, \Delta, \mathcal{D}, \sigma\left(t_{i}, x\right)\right)=\mathbb{P}\left(\exists t \in\left[t_{i}, t_{i+1}\right]: X_{t}^{N} \notin \mathcal{D} \mid X_{t_{i}}^{N}=x, X_{t_{i+1}}^{N}=y\right)
$$

as $\Delta$ goes to 0 . An asymptotic expansion for this probability is available (for smooth domain). But it is computational demanding and thus, this is of limited interest for real time computations. One may alternatively think of locally approximating the domain by a half-space (see Gobet [14]). This may be formalized as follows. For $X_{t_{i}}^{N}=x$ close to the boundary $\partial \mathcal{D}$, denote by $\pi(x)$ its projection on $\partial \mathcal{D}$ and by $n(x)$ the associated inward normal vector at 
this point. In the computation of $p_{i}(x, y, \Delta)$, then replace $\mathcal{D}$ by the half-space containing $x$ and delimited by the tangent hyper-plane at the point $\pi(x)$. Then, the conditional trigger probability becomes explicit because this boils down to a one-dimensional situation. This leads to

$$
p\left(x, y, \Delta, \mathcal{D}, \sigma\left(t_{i}, x\right)\right) \approx \begin{cases}1 & \text { if } x \text { or } y \text { are outside } \mathcal{D} \\ \exp \left(-2 \frac{d(x, \mathcal{D}) d(y, \mathcal{D})}{\left|\sigma\left(t_{i}, x\right) \cdot n(x)\right|^{2} \Delta}\right) & \text { otherwise }\end{cases}
$$

One can prove (see Gobet [14]) that the simulation bias is still of order $\Delta$, as before when the conditional trigger probability was exactly computed. But this result is valid if the domain is smooth enough, while in practice for multiple barriers the domain is of the form

$$
\mathcal{D}=] D_{1}, U_{1}[\times \cdots \times] D_{d}, U_{d}[
$$

(where $d$ is the number of assets): thus, it exhibits corners. Hence, for each asset, there is a single or double barrier for which the individual conditional trigger probability is easy to evaluate ${ }^{3}$ :

$$
\begin{aligned}
p_{i, j}(x, y, \Delta)= & \mathbb{P}\left(\exists t \in\left[t_{i}, t_{i+1}\right]: X_{j, t}^{N} \notin\right] D_{j}, U_{j}\left[\mid X_{t_{i}}^{N}=x, X_{t_{i+1}}^{N}=y\right) \\
= & \left\{\begin{array}{l}
1 \quad \text { if } x_{j} \text { or } y_{j} \text { are above } U_{j} \text { or below } D_{j}, \\
\sum_{k=-\infty}^{+\infty}\left[\exp \left(-2 \frac{k\left(U_{j}-D_{j}\right)\left(k\left(U_{j}-D_{j}\right)+y_{j}-x_{j}\right)}{\left|\sigma_{j}\left(t_{i}, x\right)\right|^{2} \Delta}\right)\right. \\
\left.-\exp \left(-2 \frac{\left(k\left(U_{j}-D_{j}\right)+x_{j}-U_{j}\right)\left(k\left(U_{j}-D_{j}\right)+y_{j}-U_{j}\right)}{\left|\sigma_{j}\left(t_{i}, x\right)\right|^{2} \Delta}\right)\right] \text { otherwise. }
\end{array}\right.
\end{aligned}
$$

If the assets are not correlated, the individual trigger events on the interval $\left[t_{i}, t_{i+1}\right]$ are independent and thus

$$
1-p\left(x, y, \Delta, \mathcal{D}, \sigma\left(t_{i}, x\right)\right)=\prod_{j=1}^{d}\left(1-p_{i, j}(x, y, \Delta)\right)
$$

But in the case of correlated assets, there is no closed formula for $p$. In that case, Shevchenko [27] suggests to use the theory of copulas (Joe [19]) in order to derive lower and an upper bounds for $p$. We recall the Frechet bounds: for any events $\left(A_{j}\right)_{1 \leq j \leq d}$, one has

$$
\left(\sum_{j=1}^{d} \mathbb{P}\left(A_{j}\right)-(d-1)\right)_{+} \leq \mathbb{P}\left(\bigcap_{1 \leq j \leq d} A_{j}\right) \leq \min _{1 \leq j \leq d} \mathbb{P}\left(A_{j}\right) .
$$

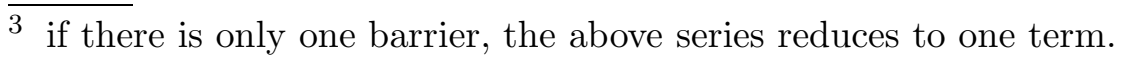


In our case, it writes

$$
\left(1-\sum_{j=1}^{d} p_{i, j}(x, y, \Delta)\right)_{+} \leq 1-p\left(x, y, \Delta, \mathcal{D}, \sigma\left(t_{i}, x\right)\right) \leq 1-\max _{1 \leq j \leq d} p_{i, j}(x, y, \Delta) .
$$

Denote by $1-p_{i}^{L}(x, y, \Delta)$ and $1-p_{i}^{U}(x, y, \Delta)$ the above lower and upper bounds. Then clearly, for positive payoff $f$ one has

$$
\begin{aligned}
& f\left(X_{T}^{N}\right) \prod_{i=0}^{N-1}\left(1-p_{i}^{L}\left(X_{t_{i}}^{N}, X_{t_{i+1}}^{N}, \Delta\right)\right) \\
& \leq f\left(X_{T}^{N}\right) \prod_{i=0}^{N-1}\left(1-p\left(X_{t_{i}}^{N}, X_{t_{i+1}}^{N}, \Delta, \mathcal{D}, \sigma\left(t_{i}, x\right)\right)\right) \\
& \leq f\left(X_{T}^{N}\right) \prod_{i=0}^{N-1}\left(1-p_{i}^{U}\left(X_{t_{i}}^{N}, X_{t_{i+1}}^{N}, \Delta\right)\right)
\end{aligned}
$$

which gives two Monte Carlo estimates of $\mathbb{E}\left(\mathbf{1}_{\forall t<T: X_{t} \in \mathcal{D}} f\left(X_{T}\right)\right)$. These are lower and upper bounds if the $\left(X_{t_{i}}\right)_{i}$ are simulated without error. In Shevchenko [27], it is proposed to take the average as an estimator.

Numerical results are brought together with the methods of barrier shifting in Section 3.

\section{Shifting the barrier}

We have mentioned in the preceding section that for the evaluation of $\mathbb{E}\left(\Psi_{T}\right)$ with $\Psi_{T}=\mathbf{1}_{\forall t<T: X_{t}>D}\left(X_{T}-K\right)_{+}$(Down Out Call), the simulation of $\Psi_{T}^{N}=$ $\mathbf{1}_{\forall t_{i}<T: X_{t_{i}}>D}\left(X_{T}-K\right)_{+}$yields a systematic overestimation, because by monitoring the process only at times $\left(t_{i}\right)_{i}$, we ignore its possible exit between these times. However, this positive bias shrinks to 0 as the number of monitoring dates $N$ goes to infinity. One natural idea to compensate the bias is to shift the barrier $D$ inside the activation zone of the option (i.e. one increases $D$ to get $D_{N}$ ) and thus to compute

$$
\Psi_{T}^{N, \text { shift }}=\mathbf{1}_{\forall t_{i}<T: X_{t_{i}}>D_{N}}\left(X_{T}-K\right)_{+} .
$$

Of course, the new barrier $D_{N}$ has to be tuned accurately, in order to exactly remove the overestimation bias. This is not a trivial issue, but the way has been paved by Broadie et al. [7] for a single asset $X$ in the Black-Scholes model with constant volatility $\sigma$. Namely, by setting

$$
D_{N}=D \exp (0.5826 \sigma \sqrt{\Delta}),
$$


one gets

$$
\mathbb{E}\left(\Psi_{T}^{N, \text { shift }}\right)-\mathbb{E}\left(\Psi_{T}\right)=o\left(\Delta^{1 / 2}\right)
$$

instead of $O\left(\Delta^{1 / 2}\right)$ without shifting the barrier. The constant $0.5826 \ldots$ is defined later in (19).

Recently in Gobet and Menozzi [18], it has been established that there is a universal rule for shifting the barrier. We discuss this hereafter. The purpose of this section is to provide several refinements about the idea of shifting the barrier, for the pricing of continuously monitored barrier option, for the pricing of discrete barrier option as well, in the case of constant and non constant volatilities, including jumps or not. Numerical experiments are postponed to Section 3.

\subsection{Understanding the influence of the monitoring frequency}

Before coming into the details of barrier shifting, it is essential to well understand what the main bias term in $\Psi_{T}^{N}-\Psi_{T}$ is.

Actually, during the last decade, it has been paid a lot of attention to the study of the associated convergence. Regarding the rate of convergence, it is proved quite generally that it is at most of order $\Delta^{1 / 2}$. Namely, for a smooth domain $\mathcal{D}$ and a general Itô process $X$, one has (see Gobet and Menozzi [17])

$$
\mathbb{E}\left(\mathbf{1}_{\forall t_{i}<T: X_{t_{i}} \in \mathcal{D}} f\left(X_{T}\right)-\mathbf{1}_{\forall t<T: X_{t} \in \mathcal{D}} f\left(X_{T}\right)\right)=O\left(\Delta^{1 / 2}\right) .
$$

In addition, for a positive payoff function $f$ and for a solution $X$ to a non degenerate SDE, the above error is bounded from below by a positive constant times $\Delta^{1 / 2}$ (see Gobet and Menozzi [16]). These theoretical results are supported by numerical experiments, which illustrate well that a discrete sampling for such exotic contracts yields a positive bias, slowly decreasing. This is analogous when in addition, an Euler scheme is used to approximate the underlying process $X$. Hence in the following, we neglect this extra error which contribution turns to be of order $\Delta$ (see Paragraph 1.3).

The key point in the error analysis is to make the connection with the asymptotics of the overshoot $Y^{N}$. The overshoot is defined by the distance of the process to the barrier (the boundary of $\mathcal{D}$ ) at the discrete trigger $\tau^{N}=\inf \left\{t_{i}\right.$ : $\left.X_{t_{i}} \notin \mathcal{D}\right\}:$

$$
Y^{N}=d\left(X_{\tau^{N}}, \partial \mathcal{D}\right)
$$

Actually, the important role of the overshoot has been underlined for years in the binomial tree methods. When the first node of the tree $\left(\log \left(X_{0}\right)+\right.$ 
$i \sigma \sqrt{\Delta})_{i}$ above the upper barrier $\log (U)$ coincides with the barrier, the overshoot of the binomial tree vanishes. In that case, one observes that the binomial tree method is quite accurate. Otherwise, the overshoot is equal to $\sigma \sqrt{\Delta}\left(1-\operatorname{Frac}\left(\frac{\log \left(U / x_{0}\right)}{\sigma \sqrt{\Delta}}\right)\right)$, where $\operatorname{Frac}(x)$ denotes the fractional part of $x$ (with the convention $\operatorname{Frac}(x)=1$ if $x \in \mathbb{N}$ ). Then, one knows that the numerical error is essentially proportional to this overshoot (see Gobet [12] for a proof) and thus creates nasty oscillations as $N$ varies: see Figure 1.

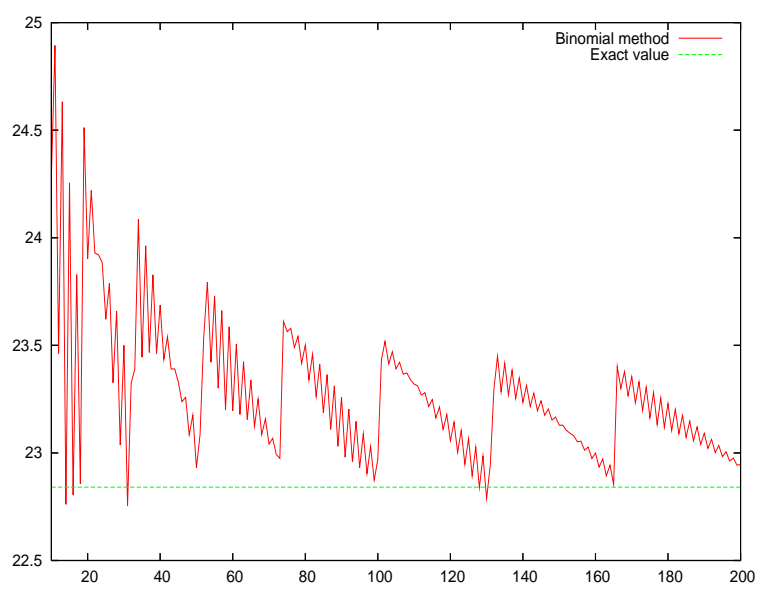

Figure 1. Up and Out Put (non discounted) with strike 900 and upper barrier at 1150. Underlying asset: initial value $=1000, T=1$ year, interest rate $=0.05$ and volatility $=0.2$.

In the general case, to decompose the error we use the process $M_{t}=\mathbb{E}\left(\mathbf{1}_{T<\tau_{t}} f\left(X_{T}\right) \mid \mathcal{F}_{t}\right)$ where $\tau_{t}=\inf \left\{s \geq t: X_{s} \notin \mathcal{D}\right\}$ is the trigger time after $t$. Clearly, if no trigger has occurred before $t$, then $M_{t}$ is the price at time $t$ of the barrier option. We collect below the main properties related to $M$ :

i) $X_{t} \notin \mathcal{D} \Longrightarrow \tau_{t}=t$ and $M_{t}=0$;

ii) for any given time $t,\left(M_{s \wedge \tau_{t}}\right)_{t \leq s \leq T}$ is a martingale ${ }^{4}$;

iii) under non degeneracy ${ }^{5}$ on $X$ and mild smoothness assumptions on $\mu, \sigma$ and $\mathcal{D}$, one has

$$
M_{t}=u\left(t, X_{t}\right)
$$

where $u$ is a smooth function on $\left[0, T\left[\times \mathcal{D}\right.\right.$, vanishing on $\left[0, T\left[\times \mathcal{D}^{c}\right.\right.$ and solving a parabolic PDE with Cauchy-Dirichlet boundary conditions: $u_{t}^{\prime}+$ $\sum_{i} \mu_{i} u_{x_{i}}^{\prime}+\frac{1}{2} \sum_{i, j}\left[\sigma \sigma^{*}\right]_{i, j} u_{x_{i}, x_{j}}^{\prime \prime}=0$ for $t<T, x \in \mathcal{D} ; u(T, x)=f(x)$ for $x \in \mathcal{D} ; u(t, x)=0$ for $x \notin D$ and $t<T$.

\footnotetext{
$\overline{4}$ indeed, one easily checks that $M_{s \wedge \tau_{t}}=\mathbf{1}_{s \leq \tau_{t}} M_{s}=\mathbb{E}\left(\mathbf{1}_{T<\tau_{t}} f\left(X_{T}\right) \mid \mathcal{F}_{s}\right)$.

5 ellipticity or hypo-ellipticity condition plus non characteristic boundary condition
} 
Then, it follows

$$
\begin{aligned}
& \mathbb{E}\left(\mathbf{1}_{\forall t_{i}<T: X_{t_{i}} \in \mathcal{D}} f\left(X_{T}\right)-\mathbf{1}_{\forall t<T: X_{t} \in \mathcal{D}} f\left(X_{T}\right)\right) \\
= & \mathbb{E}\left(M_{T \wedge \tau^{N}}-M_{0}\right)=\sum_{0 \leq i<N} \mathbb{E}\left(M_{t_{i+1} \wedge \tau^{N}}-M_{t_{i} \wedge \tau^{N}}\right) \text { (properties i)-iii)) } \\
= & \sum_{0 \leq i<N} \mathbb{E} \mathbf{1}_{t_{i}<\tau^{N}}\left(M_{t_{i+1}}-M_{t_{i}}\right) \\
= & \sum_{0 \leq i<N} \mathbb{E} \mathbf{1}_{t_{i}<\tau^{N}}\left(M_{t_{i+1}}-M_{t_{i+1} \wedge \tau_{t_{i}}}\right)+\sum_{0 \leq i<N} \mathbb{E} \mathbf{1}_{t_{i}<\tau^{N}}\left(M_{t_{i+1} \wedge \tau_{t_{i}}}-M_{t_{i}}\right) \\
= & \left.\sum_{0 \leq i<N} \mathbb{E} \mathbf{1}_{t_{i}<\tau^{N}} \mathbf{1}_{\tau_{t_{i}}<t_{i+1}}\left(M_{t_{i+1}}-M_{\tau_{t_{i}}}\right) \text { (properties i)-ii) and } \tau_{t_{i}} \geq t_{i}\right) .
\end{aligned}
$$

Because $M$ vanishes when $X$ is outside $\mathcal{D}$ and $u$ is smooth inside $\mathcal{D}$, one has $M_{t_{i+1}}-M_{\tau_{t_{i}}}=\mathbf{1}_{X_{t_{i+1}} \in \mathcal{D}} \nabla_{x} u\left(\tau_{t_{i}}, X_{\tau_{t_{i}}}\right) \cdot\left(X_{t_{i+1}}-X_{\tau_{t_{i}}}\right)$ plus a term whose expectation is of order $\Delta$ and which can be neglected (see Gobet and Menozzi [18] for details). This gives

$$
\begin{aligned}
& \mathbb{E}\left(\mathbf{1}_{\forall t_{i}<T: X_{t_{i}} \in \mathcal{D}} f\left(X_{T}\right)-\mathbf{1}_{\forall t<T: X_{t} \in \mathcal{D}} f\left(X_{T}\right)\right) \\
& =\sum_{0 \leq i<N} \mathbb{E}\left(\mathbf{1}_{t_{i}<\tau^{N}} \mathbf{1}_{\tau_{t_{i}}<t_{i+1}} \mathbb{E}\left(\mathbf{1}_{X_{t_{i+1}} \in \mathcal{D}} \nabla_{x} u\left(\tau_{t_{i}}, X_{\tau_{t_{i}}}\right) \cdot\left(X_{t_{i+1}}-X_{\tau_{t_{i}}}\right) \mid \mathcal{F}_{\tau_{t_{i}}}\right)\right)+O(\Delta) \text {. }
\end{aligned}
$$

Denote by $n(s)$ the inward normal unit vector at $s \in \partial \mathcal{D}$ and set $\partial_{n} u(t, s)=$ $\nabla_{x} u(t, s) \cdot n(s)$ : since $u=0$ on $\partial \mathcal{D}, \nabla_{x} u(t, s)$ lies only in the normal direction. In addition, with a probability exponentially close to 1 w.r.t. $N$, on $\mathcal{F}_{\tau_{t_{i}}} X_{t_{i+1}}$ is close to the boundary and the normal component of $X_{t_{i+1}}-X_{\tau_{t_{i}}}$ has an amplitude equal to $d\left(X_{t_{i+1}}, \partial \mathcal{D}\right)$. Bringing together all these remarks leads to

$$
\begin{aligned}
& \mathbb{E}\left(\mathbf{1}_{X_{t_{i+1}} \in \mathcal{D}} \nabla_{x} u\left(\tau_{t_{i}}, X_{\tau_{t_{i}}}\right) \cdot\left(X_{t_{i+1}}-X_{\tau_{t_{i}}}\right) \mid \mathcal{F}_{\tau_{t_{i}}}\right) \\
= & \partial_{n} u\left(\tau_{t_{i}}, X_{\tau_{t_{i}}}\right) \mathbb{E}\left(\mathbf{1}_{X_{t_{i+1}} \in \mathcal{D}} d\left(X_{t_{i+1}}, \partial \mathcal{D}\right) \mid \mathcal{F}_{\tau_{t_{i}}}\right)+o(\Delta) \\
= & \partial_{n} u\left(\tau_{t_{i}}, X_{\tau_{t_{i}}}\right) \mathbb{E}\left(\mathbf{1}_{X_{t_{i+1}} \notin \mathcal{D}} d\left(X_{t_{i+1}}, \partial \mathcal{D}\right) \mid \mathcal{F}_{\tau_{t_{i}}}\right)+O(\Delta) \\
= & \partial_{n} u\left(\tau_{t_{i}}, X_{\tau_{t_{i}}}\right) \mathbb{E}\left(Y^{N} \mid \mathcal{F}_{\tau_{t_{i}}}\right)+O(\Delta) .
\end{aligned}
$$

Finally we get

Theorem 1 Discrete monitoring and continuous monitoring yields a difference of prices that is proportional to the expected weighted overshoot. It writes

$$
\begin{aligned}
& \mathbb{E}\left(\mathbf{1}_{\forall t_{i}<T: X_{t_{i}} \in \mathcal{D}} f\left(X_{T}\right)-\mathbf{1}_{\forall t<T: X_{t} \in \mathcal{D}} f\left(X_{T}\right)\right) \\
= & \sum_{0 \leq i<N} \mathbb{E}\left(\mathbf{1}_{t_{i+1}=\tau^{N}} \partial_{n} u\left(\tau_{t_{i}}, X_{\tau_{t_{i}}}\right) Y^{N}\right)+O(\Delta) .
\end{aligned}
$$

Since the increments of $X$ are of order $\Delta^{1 / 2}$, the same order applies to the overshoot $Y^{N}$ and this justifies why the discrete monitoring yields a simulation 
bias of this order. More interesting is to see that an expansion at the order $\Delta^{1 / 2}$ is available, provided that the limit of the triplet $\left(\tau^{N}, X_{\tau^{N}}, \Delta^{-1 / 2} Y^{N}\right)$ can be identified. When $\left(X_{t}=x_{0}+W_{t}\right)_{t}$ is a linear Brownian motion and $\mathcal{D}=]-\infty, U$, finding the asymptotics of the renormalized overshoot $\sqrt{\frac{N}{T}} Y^{N}$ can be made as follows. Set $\left(s_{i}=\sqrt{\frac{N}{T}} W_{i T / N}\right)_{i}$, which defines a Gaussian random walk, and put $\tau^{u}=\inf \left\{i \geq 0: s_{i}>u\right\}$. Clearly one has

$$
\sqrt{\frac{N}{T}} Y^{N}:=\sqrt{\frac{N}{T}}\left(X_{\tau^{N}}-U\right)=\left.\left[s_{\tau^{u}}-u\right]\right|_{u=u_{N}}:=y\left(u_{N}\right)
$$

where $u_{N}=\sqrt{\frac{N}{T}}\left(U-x_{0}\right)$ goes to infinity with $N$. From the renewal theory (see Siegmund [28]), $s_{\tau^{u}}-u=y(u)$ weakly converges as $u$ goes to infinity to a random variable $Y$, whose cumulative function $H$ is given by:

$$
H(y):=\left(\mathbb{E}\left[s_{\tau^{0}}\right]\right)^{-1} \int_{0}^{y} \mathbb{P}\left[s_{\tau^{0}}>z\right] d z
$$

In our shifting approach, the quantity which plays a crucial role (remind of (14)) is the expected asymptotic renormalized overshoot

$$
\bar{y}(\infty)=\mathbb{E}(Y)=\lim _{u \rightarrow \infty} \mathbb{E}(y(u))=\frac{\mathbb{E}\left[s_{\tau^{0}}^{2}\right]}{2 \mathbb{E}\left[s_{\tau^{0}}\right]}=-\frac{\zeta(1 / 2)}{\sqrt{2 \pi}}=0.5826 \ldots
$$

(see Siegmund [28]). One also knows from Chang and Peres [8] that

$$
\bar{y}(0):=\mathbb{E}(y(0))=\mathbb{E}\left[s_{\tau^{0}}\right]=\frac{1}{\sqrt{2}}=0.7071 \ldots
$$

Unfortunately, the other values of $\bar{y}(u)=\mathbb{E}(y(u))$ are not known (see Appendix for a numerical approximation).

The previous results by Siegmund on the scalar Brownian motion have been recently extended to general diffusion processes (and to the associated Euler scheme as well).

Theorem 2 (Gobet and Menozzi [18]). The sequence $\left(\tau^{N}, X_{\tau^{N}}, \Delta^{-1 / 2} Y^{N}\right)_{N}$ weakly converges to $\left(\tau, X_{\tau},\left|n \sigma\left(\tau, X_{\tau}\right)\right| Y\right)$ where $Y$ is a random variable independent of $\left(\tau, X_{\tau}\right)$, and which cumulative function is equal to $H$ (given in (18)).

As a consequence and relying on (easy) extra uniform integrability results, 
one can pass to the limit in theorem 1 to get:

$$
\begin{aligned}
& \mathbb{E}\left(\mathbf{1}_{\forall t_{i}<T: X_{t_{i}} \in \mathcal{D}} f\left(X_{T}\right)-\mathbf{1}_{\forall t<T: X_{t} \in \mathcal{D}} f\left(X_{T}\right)\right) \\
= & \bar{y}(\infty) \sqrt{\frac{T}{N}} \mathbb{E}\left(\mathbf{1}_{\tau<T} \partial_{n} u\left(\tau, X_{\tau}\right)\left|n \sigma\left(\tau, X_{\tau}\right)\right|\right)+o\left(N^{-1 / 2}\right) .
\end{aligned}
$$

As required, this is an overestimation (for positive payoff $f$ ) because the inward normal derivative of $u$ is positive.

If we look carefully at the assumptions in the quoted references, the domain $\mathcal{D}$ needs to be a little smooth (of class $C^{2}$ ) and it does not allow us to directly apply the results to domains with corners. Actually, the limitation is essentially due to technical considerations related to good controls of the derivatives of $u$, solution of the PDE. In Menozzi [21], these technicalities are handled in the case of a bi-dimensional drifted Brownian motion.

As a computational consequence of this result, the Romberg extrapolation techniques can be applied to get a more accurate procedure. Namely, by using two monitoring frequencies $N / T$ and $2 N / T$ and by averaging out independent simulations of

$$
\Psi_{T}^{N, \text { Romberg }}=f\left(X_{T}\right)\left(\sqrt{2} \mathbf{1}_{\forall i \leq 2 N: X_{i T /(2 N)} \in \mathcal{D}}-\mathbf{1}_{\forall i \leq N: X_{i T / N} \in \mathcal{D}}\right) /(\sqrt{2}-1),
$$

we get

$$
\mathbb{E} \Psi_{T}^{N, \text { Romberg }}-\mathbb{E} \Psi_{T}=o\left(N^{-1 / 2}\right) .
$$

Hence, at the first sight, we get a more accurate estimation of the price. However, there are two drawbacks. First, the computational time has essentially been multiplied by a factor 3 (because one simulates with $N$ and $2 N$ time steps). Second, the variance is approximately multiplied by a factor $\left((\sqrt{2})^{2}+1^{2}\right) /(\sqrt{2}-1)^{2} \approx 17.5$, which increases the statistical error of the Monte Carlo method.

\subsection{Derivation of the sensitivity to the barriers}

As mentioned in the introduction of this section, to compensate the systematic overestimation of discrete monitoring, we may think of slightly increase the trigger zone by pushing the barrier towards the initial value of the underlying process $X$. The question is how much should we shift the barrier? The answer is strongly related to the sensitivity of the option price to the barrier.

We start with the simplest case of a drifted Brownian motion for $X_{t}=x+$ $\mu t+\sigma W_{t}$ and an upper barrier $U$. In that case, due to explicit conditional 
trigger probability one gets

$$
\begin{aligned}
& \partial_{U} \mathbb{E}\left(\mathbf{1}_{\forall t<T: X_{t}<U} f\left(X_{T}\right)\right) \\
& =\partial_{U} \int_{-\infty}^{U} f(y)\left(1-\exp \left(-2 \frac{(U-x)(U-y)}{\sigma^{2} T}\right)\right) \frac{1}{\sigma \sqrt{2 \pi T}} \exp \left(-\frac{(y-x-\mu T)^{2}}{2 \sigma^{2} T}\right) \mathrm{d} y \\
& =\int_{-\infty}^{U} f(y) \exp \left(-2 \frac{(U-x)(U-y)}{\sigma^{2} T}\right) \frac{2(2 U-x-y)}{\sigma^{3} \sqrt{2 \pi T^{3}}} \exp \left(-\frac{(y-x-\mu T)^{2}}{2 \sigma^{2} T}\right) \mathrm{d} y \\
& =\int_{-\infty}^{U} f(y) \frac{2}{\sigma^{2}} \exp \left(\frac{\mu(y-x)}{\sigma^{2}}-\frac{\mu^{2}}{2 \sigma^{2}} T\right) h^{0}(T, 2 U-x-y) \mathrm{d} y
\end{aligned}
$$

where we set

$$
h^{\mu}(t, b)=\frac{|b|}{\sqrt{2 \pi \sigma^{2} t^{3}}} \exp \left(-\frac{(b-\mu t)^{2}}{2 \sigma^{2} t}\right)=h^{0}(t, b) \exp \left(\frac{\mu b}{\sigma^{2}}-\frac{\mu^{2}}{2 \sigma^{2}} t\right) .
$$

Actually, the first hitting time $\tau$ for $X$ of the level $U$ has a density equal to $h^{\mu}(t, U-x)$ (see Karatzas and Shreve [20]). The above relation with $h^{0}$ can be also obtained using the Girsanov theorem.

For the purpose of the barrier shifting, it is more convenient to relate $\partial_{U} \mathbb{E}\left(\mathbf{1}_{\forall t<T: X_{t}<U} f\left(X_{T}\right)\right)$ to the gradient of the solution $u$ of the PDE at the barrier. For this, use the convolution equality

$$
h^{0}(T, 2 U-x-y)=\int_{0}^{T} h^{0}(t, U-x) h^{0}(T-t, U-y) \mathrm{d} t
$$

$(U>x, U>y)$ which results from the definition of $h$ and the independence of hitting times of $\left(x+\sigma W_{t}\right)_{t}$ of successive levels. Then,

$\partial_{U} \mathbb{E}\left(\mathbf{1}_{\forall t<T: X_{t}<U} f\left(X_{T}\right)\right)=$

$$
\int_{0}^{T} h^{\mu}(t, U-x) \int_{-\infty}^{U} f(y) \frac{2}{\sigma^{2}} \exp \left(\frac{\mu(y-U)}{\sigma^{2}}-\frac{\mu^{2}}{2 \sigma^{2}}(T-t)\right) h^{0}(T-t, U-y) \mathrm{d} y \mathrm{~d} t .
$$

In the same way, a direct computation shows that the above integral w.r.t. $y$ is actually equals to $-\partial_{x} u(t, U)$, which finally gives

$$
\partial_{U} \mathbb{E}\left(\mathbf{1}_{\forall t<T: X_{t}<U} f\left(X_{T}\right)\right)=-\mathbb{E}\left(\mathbf{1}_{\tau \leq T} \partial_{x} u\left(\tau, X_{\tau}\right)\right) .
$$

This formula is remarkably simple. Actually it is not a coincidence with the Brownian setting. It turns out that it can be extended to general diffusion models. More interesting is to notice that this gradient formula looks like the main term appearing in the discrete monitoring, this is discussed a bit later.

In the general case, the above explicit computations are no more explicit and we have to carry out an alternative way to proceed. We give below another proof using basic stochastic calculus arguments, which has the great advantage 
to handle a large generality of trigger zones and stochastic processes. Before, we mention that related problems have been considered in the PDE literature. Indeed, the price function is solution of Cauchy-Dirichlet PDE, of the same type than the position of a elastic structure to which external forces are applied. Hence, computing the barrier sensitivity is very analogous to compute the shape sensitivity of elastic structures (see Allaire [1] and references therein about shape optimization). Sensitivities w.r.t. the domain are classic issues in the numerical analysis literature and date back to Hadamard at the beginning of the century. Recently in Costantini et al. [10], extensions to time-dependent domains and to general diffusion processes have been obtained by probabilistic techniques. Actually, the proof is rather elementary and we give it in the previous case of an upper barrier $U$ and linear diffusion process $X$. Denote by $\tau^{\epsilon}=\inf \left\{t \geq 0: X_{t} \geq U-\epsilon\right\}$ the first hitting time of $U-\epsilon$ by $X(\epsilon>0)$. Clearly $\left(\tau^{\epsilon}\right)_{\epsilon}$ defines a increasing sequence as $\epsilon \downarrow 0$, bounded by $\tau$ and it is not hard ${ }^{6}$ to show that it also converges to $\tau$. We aim at computing the limit of $\left[\mathbb{E}\left(\mathbf{1}_{T<\tau} f\left(X_{T}\right)\right)-\mathbb{E}\left(\mathbf{1}_{T<\tau^{\epsilon}} f\left(X_{T}\right)\right)\right] / \epsilon$ as $\epsilon \downarrow 0$, which gives the left derivative ${ }^{7}$ of $\mathbb{E}\left(\mathbf{1}_{\forall t<T: X_{t}<U} f\left(X_{T}\right)\right)$ w.r.t. $U$.

Note that $\mathbb{E}\left(\mathbf{1}_{T<\tau} f\left(X_{T}\right)\right)=u(0, x)=\mathbb{E}\left(u\left(T \wedge \tau^{\epsilon}, X_{T \wedge \tau^{\epsilon}}\right)\right)$ since $\left(u\left(s \wedge \tau, X_{s \wedge \tau}\right)\right)_{s \leq T}$ is a martingale and $\tau^{\epsilon}<\tau$. In addition, $\mathbf{1}_{T<\tau^{\epsilon}} f\left(X_{T}\right)=u\left(T \wedge \tau^{\epsilon}, X_{T \wedge \tau^{\epsilon}}\right)-$ $\mathbf{1}_{\tau^{\epsilon} \leq T} u\left(\tau^{\epsilon}, X_{\tau^{\epsilon}}\right)$ because on $T<\tau^{\epsilon}, f\left(X_{T}\right)=u\left(T, X_{T}\right)\left(X_{T}<U\right)$. Writing $X_{\tau^{\epsilon}}=U-\epsilon$ and $u\left(\tau^{\epsilon}, U\right)=0$, one finally gets

$$
\frac{\mathbb{E}\left(\mathbf{1}_{T<\tau} f\left(X_{T}\right)\right)-\mathbb{E}\left(\mathbf{1}_{T<\tau^{\epsilon}} f\left(X_{T}\right)\right)}{\epsilon}=\mathbb{E}\left(\mathbf{1}_{\tau^{\epsilon} \leq T} \frac{u\left(\tau^{\epsilon}, U-\epsilon\right)-u\left(\tau^{\epsilon}, U\right)}{\epsilon}\right) .
$$

Passing to the limit, we clearly obtain the equality (24), not only for a drifted Brownian motion but for a general diffusion model for $X$.

We now state a more general result for a multidimensional domain $\mathcal{D} \subset \mathbb{R}^{d}$ and take for instance $\mathbf{1}_{\forall t<T: X_{t} \in \mathcal{D}} f\left(X_{T}\right)$ as a general form ${ }^{8}$ of the option payoff for an initial trigger zone $\mathcal{D}$ : the price function $u(t, x)$ is solution of a parabolic $\mathrm{PDE}$ as mentioned before. We allow the domain $\mathcal{D}$ to be perturbed in various directions with various amplitudes (parameterized by a function $\Theta$ and a small parameter $\epsilon$ ), which may result in a time-dependent domain in that way:

$$
\mathcal{D}_{t}^{\epsilon}=\{x: x+\epsilon \Theta(t, x) \in \mathcal{D}\} .
$$

We focus on the price for the option associated to the perturbed trigger zone:

6 one needs here to assume that the diffusion coefficient of $X$ does not vanish at the barrier.

7 the computations for the right derivative are analogous and we skip them.

8 without extra difficulties, a rebate could be paid at the trigger time and the domain may be time-dependent as well: see Costantini et al. [10] for details. 
it is equal to $\mathbb{E}\left(\mathbf{1}_{\forall t<T: X_{t} \in \mathcal{D}_{t}^{\epsilon}} f\left(X_{T}\right)\right)$. It is proved ${ }^{9}$ (under suitable mild smoothness assumptions and ellipticity condition, see Costantini et al. [10] for details) that the latter price as a function of $\epsilon$ is continuously differentiable and its derivative at $\epsilon=0$ is given by:

$$
\mathbb{E}\left[\mathbf{1}_{\tau<T} \partial_{n} u\left(\tau, X_{\tau}\right)[\Theta . n]\left(\tau, X_{\tau}\right)\right],
$$

where $n(x)$ is the inward normal unit vector at $\partial \mathcal{D}$ at the point $x$ and $\tau=$ $\inf \left\{t>0: X_{t} \notin \mathcal{D}\right\}$ is the first exit time of $\left(X_{s}\right)_{s \geq 0}$ from $\mathcal{D}$. Equivalently, this differentiability can be written as

Theorem 3 The price of knocked-out option is differentiable with respect to trigger zone and the sensitivity is defined by

$$
\begin{aligned}
\mathbb{E}\left(\mathbf{1}_{\forall t<T: X_{t}+\epsilon \theta\left(t, X_{t}\right) \in \mathcal{D}} f\left(X_{T}\right)\right) & =\mathbb{E}\left(\mathbf{1}_{\forall t<T: X_{t} \in \mathcal{D}} f\left(X_{T}\right)\right) \\
& +\epsilon \mathbb{E}\left[\mathbf{1}_{\tau<T} \partial_{n} u\left(\tau, X_{\tau}\right)[\Theta . n]\left(\tau, X_{\tau}\right)\right]+o(\epsilon) .
\end{aligned}
$$

2.3 Connecting discrete/continuous monitoring: the BArrier Shifting Techniques (BAST)

We recall that from $(21)$, one gets

$$
\begin{aligned}
& \mathbb{E}\left(\mathbf{1}_{\forall t_{i}<T: X_{t_{i}} \in \mathcal{D}} f\left(X_{T}\right)-\mathbf{1}_{\forall t<T: X_{t} \in \mathcal{D}} f\left(X_{T}\right)\right) \\
= & \bar{y}(\infty) \sqrt{\frac{T}{N}} \mathbb{E}\left(\mathbf{1}_{\tau<T} \partial_{n} u\left(\tau, X_{\tau}\right)\left|n \sigma\left(\tau, X_{\tau}\right)\right|\right)+o\left(N^{-1 / 2}\right) .
\end{aligned}
$$

Thus, combining this with (25) for the choice $\theta(t, x)=\bar{y}(\infty)|n \sigma(t, x)| n(x)$ and $\epsilon=\sqrt{\frac{T}{N}}$, one obtains several simple relations (Theorem $\left.4,5,6\right)$ between prices of discrete and continuous monitored barrier options with adjusted trigger zones. In the following, we refer to these results as BAST (BArrier Shifting Techniques).

Theorem 4 (From Discrete to Continuous (D2C)) The price of a discrete barrier option equals that of a continuous barrier option with shifted barrier:

$$
\begin{aligned}
\mathbb{E}\left(\mathbf{1}_{\forall t_{i}<T: X_{t_{i}} \in \mathcal{D}} f\left(X_{T}\right)\right) & -\mathbb{E}\left(\mathbf{1}_{\forall t<T: X_{t}+\bar{y}(\infty)|n \sigma| n\left(t, X_{t}\right) \sqrt{\frac{T}{N}} \in \mathcal{D}} f\left(X_{T}\right)\right) \\
& =o\left(N^{-1 / 2}\right) .
\end{aligned}
$$

9 the arguments are similar to those used before for the case of one-dimensional barrier $U$. 
In the above expression, $n(x)$ has to be understood as the inward normal to $\partial \mathcal{D}$ at the closest point to $x$ on $\partial \mathcal{D}$ (i.e. its projection on the boundary).

The above Theorem 4 is an extension of results by Broadie et al. [7] to multiassets and multi-barriers options. Such equality is useful to numerically evaluate the price of discrete barrier options only in situations where we are able to compute more efficiently the price of continuous ones.

- This is the case for linear arithmetic/geometric Brownian motion with one single/double barrier, for which there is a closed formula which is instantaneous to compute. This is the idea developed in Broadie et al. [7]. For instance, for the Down and Out Call in a geometric Brownian motion model with volatility $\sigma$ and with dividend rate $q$, one has

$$
\begin{aligned}
\operatorname{DOC}\left(X_{0}, D\right) & =\mathbb{E}\left(e^{-r T} \mathbf{1}_{\forall t<T: X_{t}>D}\left(X_{T}-K\right)_{+}\right) \\
& =\operatorname{Call}_{B S}\left(X_{0}\right)-\left(\frac{X_{0}}{D}\right)^{1-2 \frac{(r-q)}{\sigma^{2}}} \operatorname{Call}_{B S}\left(\frac{D^{2}}{X_{0}}\right)
\end{aligned}
$$

where $\operatorname{Call}_{B S}\left(X_{0}\right)$ is the usual Black-Scholes price of the vanilla Call when the initial spot equals $X_{0}$. In that case,

$$
X_{t}+\bar{y}(\infty)|n \sigma| n\left(t, X_{t}\right) \sqrt{\frac{T}{N}} \in \mathcal{D} \Longleftrightarrow X_{t}+\bar{y}(\infty) \sigma X_{t} \sqrt{\frac{T}{N}}>D,
$$

which is equivalent (at the order $\left.o\left(N^{-1 / 2}\right)\right)$ to

$$
X_{t}>D \exp \left(-\bar{y}(\infty) \sigma \sqrt{\frac{T}{N}}\right) .
$$

If we denote by $\operatorname{DOC}_{N}\left(X_{0}, D\right)$ the price of the similar DOC with $N$ monitoring dates, we get the approximation (see Broadie et al. [7])

$$
\operatorname{DOC}_{N}\left(X_{0}, D\right)=\operatorname{DOC}\left(X_{0}, D \exp \left(-\bar{y}(\infty) \sigma \sqrt{\frac{T}{N}}\right)\right)+o\left(N^{-1 / 2}\right) .
$$

In the next paragraph, we discuss this approximation and we give a simple additional improvement when $X_{0}$ is close to the barrier or when $N$ is small.

- This may also be applied if we can perform a efficient Monte Carlo methods for the continuous barrier option, using for instance the Brownian bridge techniques (see paragraph 1). To illustrate this, consider again an Down and Out Call with a Merton's model ${ }^{10}$ of type (6). Then, the payoff (7)

\footnotetext{
${ }^{10}$ in this jump diffusion model, the previous discussion is not supported by theoretical results. Nevertheless, the intuition is clear. Indeed, if the option is triggered
} 
(written with a lower barrier $D$ ) has to be replaced by

$$
\begin{aligned}
\left(X_{T}-K\right)_{+} & \left(1-p\left(X_{\tau_{k}}, X_{T}, T-\tau_{k}, D \exp \left(-\bar{y}(\infty) \sigma \sqrt{\frac{T}{N}}\right),|\sigma|\right)\right) \\
& \times \prod_{i=0}^{k-1}\left(1-p\left(X_{\tau_{i}}, X_{\tau_{i+1}^{-}}, \tau_{i+1}-\tau_{i}, D \exp \left(-\bar{y}(\infty) \sigma \sqrt{\frac{T}{N}}\right),|\sigma|\right)\right) .
\end{aligned}
$$

In the same way one can go from the Discrete to Continuous barrier pricing, we can approximate continuous barrier option using discrete ones with a Barrier shifting procedure.

Theorem 5 (From Continuous to Discrete (C2D)) The price of a continuous barrier option equals that of a discrete barrier option with shifted barrier:

$$
\begin{aligned}
\mathbb{E}\left(\mathbf{1}_{\forall t<T: X_{t} \in \mathcal{D}} f\left(X_{T}\right)\right) & -\mathbb{E}\left(\mathbf{1}_{\forall t_{i}<T: X_{t_{i}}-\bar{y}(\infty)|n \sigma| n\left(t_{i}, X_{t_{i}}\right) \sqrt{\frac{T}{N} \in \mathcal{D}}} f\left(X_{T}\right)\right) \\
& =o\left(N^{-1 / 2}\right) .
\end{aligned}
$$

This procedure avoids using Brownian bridge techniques and related variants. This is particularly interesting for multi-assets and multi-barriers contracts where the computation of joint trigger probabilities is not possible (see the discussion in paragraph 1.3).

Also, for contracts with a large number of monitoring dates $N_{1} \gg 1$ (daily for instance) we approximate them by the similar one with a medium number of monitoring dates $1 \ll N_{2} \ll N_{1}$, by suitably adjusting the barrier twice. This saves computational time because it is essentially linear with the number of dates.

Theorem 6 (From Discrete to Discrete (D2D)) Two discrete barrier options with two different monitoring dates $\left(t_{1, i}=i T / N_{1}\right)_{i}$ and $\left(t_{2, i}=i T / N_{2}\right)_{i}$ are equal provided that barriers are conveniently shifted:

$$
\begin{aligned}
\mathbb{E}\left(\mathbf{1}_{\forall t_{1, i}<T: X_{t_{1, i}} \in \mathcal{D}} f\left(X_{T}\right)\right) & -\mathbb{E}\left(\mathbf{1}_{\forall t_{2, i}<T: X_{t_{2, i}}+\bar{y}(\infty)|n \sigma| n\left(t_{2, i}, X_{t_{2, i}}\right)}\left(\sqrt{\frac{T}{N_{1}}}-\sqrt{\frac{T}{N_{2}}}\right) \in \mathcal{D}\right. \\
= & f\left(N_{2}^{-1 / 2}\right) .
\end{aligned}
$$

when a jump occurs, the monitoring frequency has no influence in these aspects. Hence, the link between discrete and continuous barrier options really relies on the good understanding of the Brownian part, as we have done before. 


\begin{tabular}{|c|c|c|c|c|}
\hline$N$ & Barrier $D$ & True value & $\begin{array}{l}\text { Continuous } \\
\text { monitoring }\end{array}$ & $\begin{array}{l}\text { Continuous monitoring } \\
\text { with BAST (and rela- } \\
\text { tive error in \%) }\end{array}$ \\
\hline & 87 & 6.281 & 6.244 & $6.281(0.0 \%)$ \\
\hline \multirow[t]{4}{*}{50} & 91 & 5.977 & 5.808 & $5.977(0.0 \%)$ \\
\hline & 95 & 4.907 & 4.398 & $4.907(0.0 \%)$ \\
\hline & 99 & 2.337 & 1.171 & $2.271(-2.8 \%)$ \\
\hline & 87 & 6.292 & 6.244 & $6.293(0.0 \%)$ \\
\hline \multirow[t]{4}{*}{25} & 91 & 6.032 & 5.808 & $6.033(0.0 \%)$ \\
\hline & 95 & 5.081 & 4.398 & $5.084(0.0 \%)$ \\
\hline & 99 & 2.813 & 1.171 & $2.673(-5.0 \%)$ \\
\hline & 91 & 6.187 & 5.808 & $6.194(0.1 \%)$ \\
\hline \multirow[t]{3}{*}{5} & 95 & 5.671 & 4.398 & $5.646(-0.5 \%)$ \\
\hline & 97 & 5.167 & 3.060 & $5.028(-2.7 \%)$ \\
\hline & 99 & 4.489 & 1.171 & $4.053(-9.8 \%)$ \\
\hline
\end{tabular}

Table 1

D2C approximation for the discrete DOC in a Black-Scholes model with volatility $\sigma=0.3$, interest rate $r=0.1, K=X_{0}=100, T=0.2 . N=50, N=25$ or $N=5$.

Before presenting several numerical illustrations of BAST, we provide an additional improvement when the processes are close to the barrier or when the number of dates $N$ are relatively small.

To understand the necessity of this improvement, we report few numerical values borrowed from Broadie et al. [7]. It deals with the price of discrete DOC option using the closed formula for the continuous one in a Black-Scholes model (see Formula (28)). One should carefully look at the accuracy of the approximation when the barrier $D$ varies and the number $N$ of dates as well. On the one hand, the approximation performs very well for large $N$ or for barriers $D$ far from the initial spot $X_{0}$. On the other hand, in the other cases it can be quite bad and may provide errors or order of $5 \%$ and even more.

By a careful look at the proof of the influence of the discrete monitoring (which is summed up in Equality (21)), one notices that the the crucial quantity for 
the limit is the expected overshoot

$$
\mathbb{E}\left(Y^{N}\right)=\sqrt{\frac{T}{N}} \mathbb{E}\left(y\left(u_{N}\right)\right)=\sqrt{\frac{T}{N}} \bar{y}\left(u_{N}\right)
$$

when the level (directly expressed in the logarithmic variables) is defined by $u_{N}=\left(\log \left(X_{0}\right)-\log (D)\right) /\left(\sigma \sqrt{\frac{T}{N}}\right)$. Since usually $u_{N}$ is very large (since $N$ is large and $D$ is not close to $X_{0}$ ), we may focus only on the asymptotic value $\bar{y}(\infty) \approx 0.5826$ as in Broadie et al. [6]. Indeed, in those cases, we have observed a very good performance of the BAST. However, for medium ${ }^{11}$ values of $u_{N}$, one should replace $\bar{y}(\infty)$ by $\bar{y}\left(u_{N}\right)$. In the above case of a discrete DOC, this means that Equality (28) becomes

$\operatorname{DOC}_{N}\left(X_{0}, D\right)=\operatorname{DOC}\left(X_{0}, D \exp \left[-\bar{y}\left(\log \left(X_{0} / D\right) /(\sigma \sqrt{T / N})\right) \sigma \sqrt{\frac{T}{N}}\right]\right)+o\left(N^{-1 / 2}\right)$

This is what we call in the following Adjusted BAST (ABAST). The additional problem that one has to solve is the computation of the function $u \mapsto \bar{y}(u)$, which is not obvious because only the values in 0 and $\infty$ are known. A numerical study (reported in Appendix) shows that one has the equality

$$
\bar{y}(u) \approx 0.5826+0.1245 \exp \left(-2.7 u^{1.2}\right)
$$

with less than 1 percent error. We present in Table 2 the same results as before, including now the ABAST. In this table, we also provide the value of $u_{N}$. In view of (33), there is no additional improvement in the case $u_{N}>1$ (but in this case, the approximation is already very accurate), which is confirmed by the numerical experiments. In the converse case, the improvement is significant and leads to very good results when $N$ is not small $(N=25$ and $N=50)$; the accuracy is now robust in the distance of the spot to the barrier. The improvement is not so spectacular for $N=5$, but this is not surprising because all the analysis related to barrier sensitivities and the influence of the discrete monitoring is based on the asymptotics $T / N \rightarrow 0$.

\section{$3 \quad$ Numerical tests}

We conclude this work by presenting different illustrations of the Brownian bridge techniques and of ABAST on practical examples.

${ }^{11}$ in other words, the convergence stated in (21) is not uniform in $X_{0}$. 


\begin{tabular}{|c|c|c|c|c|c|}
\hline$N$ & Barrier $D$ & True value & $\begin{array}{l}\text { BAST (and rela- } \\
\text { tive error in } \% \text { ) }\end{array}$ & $u_{N}$ & $\begin{array}{l}\text { ABAST (and rel- } \\
\text { ative error in \%) }\end{array}$ \\
\hline & 87 & 6.281 & $6.281(0.0 \%)$ & 7.33 & $6.281(0.0 \%)$ \\
\hline \multirow[t]{4}{*}{50} & 91 & 5.977 & $5.977(0.0 \%)$ & 4.97 & $5.977(0.0 \%)$ \\
\hline & 95 & 4.907 & $4.907(0.0 \%)$ & 2.70 & $4.907(0.0 \%)$ \\
\hline & 99 & 2.337 & $2.271(-2.8 \%)$ & 0.53 & $2.332(-0.2 \%)$ \\
\hline & 87 & 6.292 & $6.293(0.0 \%)$ & 5.18 & $6.281(0.0 \%)$ \\
\hline \multirow[t]{4}{*}{25} & 91 & 6.032 & $6.033(0.0 \%)$ & 3.51 & $6.033(0.0 \%)$ \\
\hline & 95 & 5.081 & $5.084(0.0 \%)$ & 1.91 & $5.0841(0.0 \%)$ \\
\hline & 99 & 2.813 & $2.673(-5.0 \%)$ & 0.37 & $2.794(-0.7 \%)$ \\
\hline & 91 & 6.187 & $6.194(0.1 \%)$ & 1.57 & $6.194(0.0 \%)$ \\
\hline \multirow[t]{3}{*}{5} & 95 & 5.671 & $5.646(-0.5 \%)$ & 0.85 & $5.663(-0.1 \%)$ \\
\hline & 97 & 5.167 & $5.028(-2.7 \%)$ & 0.51 & $5.111(-1.1 \%)$ \\
\hline & 99 & 4.489 & $4.053(-9.8 \%)$ & 0.17 & $4.353(-3.1 \%)$ \\
\hline
\end{tabular}

Table 2

D2C approximation for the discrete DOC in a Black-Scholes model with volatility $\sigma=0.3$, interest rate $r=0.1, K=X_{0}=100, T=0.2 . N=50, N=25$ or $N=5$.

\subsection{Merton's model}

Consider first the case of discrete DOC (with $N$ monitoring dates), for which we apply the D2C approximation described in (29) (with modifying $\bar{y}(\infty)$ as indicated before). The advantage is mainly computational because instead of simulating the underlying process at the $N$ monitoring dates and at the $N_{J}$ jump times ${ }^{12}$, the formula (29) requires the knowledge of the underlying process at the $N_{J}$ jump times plus the maturity $T$. In the following example, we take $\lambda=1$ and $T=0.5$, which requires in average to simulate the process at $0.5+1$ dates instead of $N+2$ dates. This can be much faster. To complete our description, we numerically observe that the confidence interval width is not significantly modified. We report numerical results in Table 3, when the barrier and $N$ vary. It turns out that, up to the statistical error due to the Monte Carlo simulations, we get a very good accuracy for high frequency monitoring ( $N$ large), exactly when such procedure may be interesting to save computational time. In Table 3, analogous results are given for a larger jump

12 which, in any case, is necessary for the simulation procedure. 


\begin{tabular}{lllll}
\hline & Barrier $D$ & $\begin{array}{l}\text { Discrete DOC } \\
\text { (true value })\end{array}$ & $\begin{array}{l}\text { Continuous DOC } \\
\text { with ABAST }\end{array}$ & $\begin{array}{l}\text { Error } \\
\text { (in percent) }\end{array}$ \\
\hline \hline 89 & $9.65(0.035)$ & $9.68(0.035)$ & 0.3 \\
& 91 & $9.02(0.035)$ & $9.05(0.034)$ & 0.3 \\
& 93 & $8.16(0.034)$ & $8.20(0.033)$ & 0.5 \\
25 & 95 & $7.04(0.033)$ & $7.08(0.032)$ & 0.6 \\
& 97 & $5.69(0.030)$ & $5.67(0.029)$ & -0.4 \\
& 99 & $4.21(0.027)$ & $3.93(0.024)$ & -6.6 \\
\hline & 89 & $9.32(0.035)$ & $9.33(0.035)$ & 0.1 \\
& 91 & $8.55(0.034)$ & $8.56(0.034)$ & 0.1 \\
& 93 & $7.52(0.033)$ & $7.53(0.033)$ & 0.1 \\
125 & 95 & $6.22(0.031)$ & $6.22(0.031)$ & 0.0 \\
97 & $4.55(0.028)$ & $4.56(0.027)$ & 0.2 \\
99 & $2.60(0.022)$ & $2.55(0.020)$ & -1.9 \\
\hline
\end{tabular}

Table 3

D2C approximation for the discrete DOC in a Merton model. Volatility $\sigma=0.3$; lognormal jumps with mean $\mu_{J}=-0.02$, standard deviation $\sigma_{J}=0.2$ and intensity $\lambda=1$; interest rate $r=0.05, K=X_{0}=100, T=0.5 . N=25$ (weekly) or $N=125$ (daily). 1000000 simulations.

The true value is obtained using Monte Carlo simulations. Values in parentheses indicate the half-width of the $95 \%$ confidence interval.

intensity $(\lambda=4)$. This is still satisfactory.

\subsection{Up and Out Put in the Heston stochastic volatility model}

In this one asset example, one considers the payoff of a continuous Up and Out Put option

$$
\mathbf{1}_{\forall t \leq T: X_{t}<U}\left(K-X_{T}\right)_{+},
$$

where the underlying asset (under the valuation probability) is defined by a Heston model

$$
\begin{aligned}
& \frac{\mathrm{d} X_{t}}{X_{t}}=r \mathrm{~d} t+\sqrt{V_{t}} \mathrm{~d} W_{t}, \\
& \mathrm{~d} V_{t}=\beta\left(\alpha-V_{t}\right) \mathrm{d} t+\sigma_{V} \sqrt{V_{t}} \mathrm{~d} B_{t},
\end{aligned}
$$




\begin{tabular}{lllll}
\hline \hline$N$ & Barrier $D$ & $\begin{array}{l}\text { Discrete DOC } \\
\text { (true value) }\end{array}$ & $\begin{array}{l}\text { Continuous DOC } \\
\text { with ABAST }\end{array}$ & $\begin{array}{l}\text { Error } \\
\text { (in percent) }\end{array}$ \\
\hline \hline 89 & $11.84(0.050)$ & $11.93(0.050)$ & 0.8 \\
& 91 & $10.99(0.049)$ & $11.10(0.049)$ & 1.0 \\
& 93 & $9.90(0.048)$ & $10.03(0.047)$ & 1.3 \\
25 & 95 & $8.52(0.045)$ & $8.68(0.044)$ & 1.9 \\
& 97 & $6.89(0.041)$ & $6.98(0.040)$ & 1.3 \\
& 99 & $5.05(0.037)$ & $4.89(0.033)$ & -3.2 \\
\hline 89 & $11.49(0.050)$ & $11.51(0.050)$ & 0.2 \\
91 & $10.50(0.049)$ & $10.52(0.049)$ & 0.2 \\
& 93 & $9.22(0.047)$ & $9.26(0.046)$ & 0.4 \\
125 & 95 & $7.62(0.044)$ & $7.65(0.043)$ & 0.4 \\
97 & $5.63(0.039)$ & $5.67(0.038)$ & 0.7 \\
99 & $3.27(0.030)$ & $3.22(0.028)$ & -1.5 \\
\hline
\end{tabular}

Table 4

Similar to Table (3) with jump intensity $\lambda=4$.

where $B$ and $W$ are two correlated Brownian motions (with correlation $\rho=$ 0.1 ). For the next experiments, we take $X_{0}=40, K=42, r=0.03$ for the interest rate, $T=0.5$ for the expiration date, and the following values for the stochastic volatility: $\beta=4, \alpha=0.04=V_{0}, \sigma_{V}=0.15$.

We apply here the C2D approximation given in Theorem 5. For the simulation of the volatility component, we use the symmetrized Euler scheme with step size $h$, which writes $V_{(i+1) h}=\left|V_{i h}+\beta\left(\alpha-V_{i h}\right) h+\sigma_{V} \sqrt{V_{i h}}\left(B_{(i+1) h}-B_{i h}\right)\right|$. Since we focus on the monitoring error, we choose a small step size $h=1 / 250$ (daily) to ensure that the related discretization error can be neglected. In the pseudo-code below, we only give the details related to the trigger, once the asset and its volatility are generated. 


\section{PSEUDO-CODE}

Delta $=\mathrm{T} / \mathrm{N}$;

// Brownian bridge techniques

prob=1;

for $i=0$ to $N-1$

prob=prob* (1-p(X [i] , X [i+1], Delta, U, $\operatorname{sqrt}(\mathrm{V}[\mathrm{i}])))$;

payof $f$ BrownianBridge $=\operatorname{prob} * \max (0, \mathrm{~K}-\mathrm{X}[\mathrm{N}])$;

// ABAST

exitABAST $=0$;

cste_ABAST=y_bar $(\log (\mathrm{U} / \mathrm{X}[0]) / \operatorname{sqrt}(\mathrm{V}[0] * \operatorname{Delta}))$;

for $i=0$ to $N-1$

U_ABAST $=U * \exp \left(-\operatorname{cste} \_A B A S T * \operatorname{sqrt}(\mathrm{V}[\mathrm{i}] * \operatorname{Delta})\right)$;

if ( $\left.\mathrm{X}[\mathrm{i}+1]>\mathrm{U}_{-} \mathrm{ABAST}\right)$ exitABAST $=1$;

if (exitABAST==1) payof $f$ ABAST $=0$;

else payoffABAST $=\max (0, \mathrm{~K}-\mathrm{X}[\mathrm{N}])$;

We observe that the Brownian bridge techniques still yield very accurate results w.r.t. the number of monitoring dates $N$. Indeed, for each given barrier $U$, the output values are almost constant w.r.t. $N$. As usual, the discrete monitoring procedure provides a price overestimation, which becomes larger and larger as the trigger probability ( $U$ closer to the initial spot). Regarding ABAST, the accuracy is very good, except for $U=41$ where the asymptotics is recovered only for large values of $N$. In this example of one single barrier on one single asset, the procedure using Brownian bridges is more efficient.

\subsection{Down Out Call on three assets}

We borrow this example to Shevchenko [27]. The buyer of the contract receives at $T$ the payoff $\left(X_{1, T}-K\right)_{+}$, i.e. a Call on the first asset, with knocked out lower barriers on the three assets. The assets are modelled by correlated geometric Brownian motions with constant coefficients. Our Table 6 (which completes Table 4 in Shevchenko [27]) compares 4 estimators: 1) using the simple discrete monitoring; 2 and 3) using the lower and upper copula bounds to compute the conditional trigger probability (see inequalities (12-13)); 4) using ABAST. In the latter case, according to Theorem 5, each barrier are shifted separately: the lower barrier for the asset $X_{k}$ becomes $D_{k} \exp \left(\bar{y}\left(u_{k, N}\right) \sigma_{k} \sqrt{\frac{T}{N}}\right)$ where $u_{k, N}=$ $\log \left(X_{k, 0} / D_{k}\right) /\left(\sigma_{k} \sqrt{\frac{T}{N}}\right)$. Contrary to the Brownian bridge techniques, we do not need to take into account the correlation between assets to adjust the barriers. This theoretical choice is confirmed by numerical experiments in Table 6 and 7. 


\begin{tabular}{|c|c|c|c|c|}
\hline $\begin{array}{l}\text { Barrier } \\
U\end{array}$ & $\begin{array}{l}\text { Discrete UOP } \\
/ \text { Brownian } \\
\text { Bridge } \\
\text { ABAST } \\
(N=13)\end{array}$ & $\begin{array}{l}\text { Discrete UOP } \\
/ \text { Brownian } \\
\text { Bridge } \\
\text { ABAST } \\
(N=26)\end{array}$ & $\begin{array}{l}\text { Discrete UOP } \\
/ \text { Brownian } \\
\text { Bridge } \\
\text { ABAST } \\
(N=52)\end{array}$ & $\begin{array}{l}\text { Discrete UOP } \\
/ \text { Brownian } \\
\text { Bridge } \\
\text { ABAST } \\
(N=126)\end{array}$ \\
\hline 41 & $\begin{array}{l}1.729 \\
0.984 \\
1.133\end{array}$ & $\begin{array}{l}1.516 \\
0.984 \\
1.040\end{array}$ & $\begin{array}{l}1.364 \\
0.983 \\
0.995\end{array}$ & $\begin{array}{l}1.233 \\
0.983 \\
0.982\end{array}$ \\
\hline 42 & $\begin{array}{l}2.244 \\
1.730 \\
1.752\end{array}$ & $\begin{array}{l}2.110 \\
1.728 \\
1.726\end{array}$ & $\begin{array}{l}2.008 \\
1.727 \\
1.725\end{array}$ & $\begin{array}{l}1.913 \\
1.727 \\
1.727\end{array}$ \\
\hline 43 & $\begin{array}{l}2.595 \\
2.253 \\
2.251\end{array}$ & $\begin{array}{l}2.510 \\
2.251 \\
2.250\end{array}$ & $\begin{array}{l}2.442 \\
2.250 \\
2.250\end{array}$ & $\begin{array}{l}2.378 \\
2.250 \\
2.250\end{array}$ \\
\hline 44 & $\begin{array}{l}2.808 \\
2.594 \\
2.591\end{array}$ & $\begin{array}{l}2.756 \\
2.593 \\
2.592\end{array}$ & $\begin{array}{l}2.714 \\
2.592 \\
2.592\end{array}$ & $\begin{array}{l}2.674 \\
2.592 \\
2.592\end{array}$ \\
\hline 45 & $\begin{array}{l}2.926 \\
2.802 \\
2.800\end{array}$ & $\begin{array}{l}2.897 \\
2.801 \\
2.801\end{array}$ & $\begin{array}{l}2.873 \\
2.801 \\
2.801\end{array}$ & $\begin{array}{l}2.850 \\
2.801 \\
2.801\end{array}$ \\
\hline 46 & $\begin{array}{l}2.988 \\
2.920 \\
2.920\end{array}$ & $\begin{array}{l}2.973 \\
2.920 \\
2.920\end{array}$ & $\begin{array}{l}2.960 \\
2.920 \\
2.919\end{array}$ & $\begin{array}{l}2.948 \\
2.920 \\
2.920\end{array}$ \\
\hline
\end{tabular}

Table 5

Continuous UOP in a Heston model with parameters: $X_{0}=40, K=42, r=0.03$, $T=0.5, \beta=4, \alpha=0.04=V_{0}, \sigma_{V}=0.15, \rho=0.1$. Upper barriers from $U=41$ to $U=46$. Comparison of discrete monitoring, Brownian bridge techniques, ABAST (C2D approximation), as $N$ increases: $N=13, N=26$ (weekly), $N=52, N=126$ (daily). 10000000 simulations: the 95\%-statistical errors are smaller than 0.002 and are not reported.

It is observed in Shevchenko [27] that the spread between the prices given by upper and lower copula bounds shrinks to 0 as $N$ goes to infinity. In Table 6 , indeed it is small and it is of order of the statistical error. The ABAST behaves also very well.

In the next experiments in Table 7 , we take the barriers closer to the initial spot. In that case, the upper and lower copula bounds have a more significant impact on the price spread. Note that the larger the correlation is, the larger the spread is. This would be even worse if we took $D_{k} \approx X_{k, 0}$. The accuracy 


\begin{tabular}{cllll}
\hline $\mathrm{N}$ & $\begin{array}{l}\text { Discrete } \\
\text { monitoring }\end{array}$ & $\begin{array}{l}\text { Copula } \\
\text { Lower } \\
\text { bound }\end{array}$ & $\begin{array}{l}\text { Copula Up- } \\
\text { per bound }\end{array}$ & ABAST \\
\hline \hline 8 & $10.68(0.03)$ & $7.48(0.02)$ & $7.66(0.02)$ & $7.53(0.02)$ \\
16 & $9.85(0.03)$ & $7.53(0.02)$ & $7.59(0.02)$ & $7.52(0.02)$ \\
32 & $9.22(0.03)$ & $7.55(0.02)$ & $7.57(0.02)$ & $7.54(0.02)$ \\
64 & $8.74(0.03)$ & $7.54(0.02)$ & $7.55(0.02)$ & $7.53(0.02)$ \\
128 & $8.41(0.03)$ & $7.55(0.02)$ & $7.55(0.02)$ & $7.55(0.02)$ \\
\hline
\end{tabular}

Down Out Call on three assets. $\sigma_{1}=\sigma_{2}=\sigma_{3}=0.4, \rho_{i, j}=\rho=0.5$ for $i \neq j$, $X_{1,0}=X_{2,0}=X_{2,0}=100, K=100, r=0.05, T=1$. Barrier down: $D_{1}=D_{2}=$ $D_{3}=80$. Comparison of discrete monitoring, Brownian bridge techniques with lower and upper copula bounds, ABAST (C2D approximation), as $N$ increases. 4000000 simulations. Values in parentheses indicate the half-width of the $95 \%$ confidence interval.

of ABAST does not seem to be affected by the correlation changes.

\subsection{Down Out Call on a basket}

In this paragraph, we consider a DOC with two barriers, but the two barriers are applied to a basket of 6 assets. The payoff is given by $\mathbf{1}_{\forall t<T: B_{1, t}>D_{1}, B_{2, t}>D_{2}}\left(B_{1, T}-\right.$ $K)_{+}$where the two baskets are given by

$$
B_{1, t}=0.5 X_{1, t}+0.3 X_{2, t}+0.2 X_{3, t} \text { and } B_{2, t}=\left(X_{4, t}+X_{5, t}+X_{6, t}\right) / 3
$$

In the following, the 6 assets $X_{k}$ are modeled by correlated geometric Brownian motions, with constant correlation $\rho=0.4$ and constant volatilities $\sigma_{k}=0.3$. Their initial values are all equal to 100 . The option expiration is $T=1$ year, the interest rate equals $r=0.05$. The barriers $D_{1}=D_{2}=D$ are taken to 85, 90 and 95. During the ABAST simulation procedure, these barriers are shifted to $D_{k} \exp \left(\bar{y}\left(u_{k, N}\right) \sigma_{k, t_{i}} \sqrt{\frac{T}{N}}\right)$ where we should take for $\sigma_{k, t_{i}}$ the volatility of the basket $B_{k}$ at time $t_{i}$. Since the basket does not follow a geometric Brownian motion dynamics, the determination of $\sigma_{k, t_{i}}$ has to be made carefully. For this, consider a general Basket on different assets $X_{i}$ with weights $p_{i}$ :

$$
B_{t}=\sum_{i} p_{i} X_{i, t}
$$




\begin{tabular}{|c|c|c|c|c|c|}
\hline$\rho$ & $\mathrm{N}$ & $\begin{array}{l}\text { Discrete } \\
\text { monitoring }\end{array}$ & $\begin{array}{l}\text { Copula } \\
\text { Lower bound }\end{array}$ & $\begin{array}{l}\text { Copula Up- } \\
\text { per bound }\end{array}$ & $\mathrm{ABAST}$ \\
\hline \multirow[t]{4}{*}{0} & 16 & $1.124(0.009)$ & $0.347(0.005)$ & $0.383(0.005)$ & $0.385(0.005)$ \\
\hline & 32 & $0.855(0.008)$ & $0.352(0.005)$ & $0.362(0.005)$ & $0.357(0.005)$ \\
\hline & 64 & $0.688(0.007)$ & $0.359(0.005)$ & $0.361(0.005)$ & $0.357(0.005)$ \\
\hline & 128 & $0.579(0.007)$ & $0.359(0.005)$ & $0.360(0.005)$ & $0.359(0.005)$ \\
\hline \multirow[t]{4}{*}{0.25} & 16 & $2.73(0.02)$ & $1.12(0.01)$ & $1.22(0.01)$ & $1.24(0.01)$ \\
\hline & 32 & $2.21(0.01)$ & $1.15(0.01)$ & $1.18(0.01)$ & $1.17(0.01)$ \\
\hline & 64 & $1.87(0.01)$ & $1.15(0.01)$ & $1.16(0.01)$ & $1.15(0.01)$ \\
\hline & 128 & $1.66(0.01)$ & $1.16(0.01)$ & $1.16(0.01)$ & $1.16(0.01)$ \\
\hline \multirow[t]{4}{*}{0.5} & 16 & $4.98(0.02)$ & $2.45(0.01)$ & $2.67(0.01)$ & $2.72(0.02)$ \\
\hline & 32 & $4.22(0.02)$ & $2.52(0.01)$ & $2.59(0.01)$ & $2.57(0.02)$ \\
\hline & 64 & $3.71(0.02)$ & $2.54(0.01)$ & $2.56(0.01)$ & $2.54(0.02)$ \\
\hline & 128 & $3.39(0.02)$ & $2.56(0.01)$ & $2.56(0.01)$ & $2.56(0.02)$ \\
\hline \multirow[t]{4}{*}{0.75} & 16 & $7.93(0.02)$ & $4.45(0.02)$ & $4.89(0.02)$ & $5.01(0.02)$ \\
\hline & 32 & $6.99(0.02)$ & $4.64(0.02)$ & $4.81(0.02)$ & $4.79(0.02)$ \\
\hline & 64 & $6.33(0.02)$ & $4.69(0.02)$ & $4.76(0.02)$ & $4.73(0.02)$ \\
\hline & 128 & $5.89(0.02)$ & $4.72(0.02)$ & $4.75(0.02)$ & $4.74(0.02)$ \\
\hline \multirow[t]{4}{*}{1} & 16 & $13.02(0.03)$ & $7.70(0.02)$ & $9.71(0.03)$ & $10.02(0.03)$ \\
\hline & 32 & $12.15(0.03)$ & $8.39(0.02)$ & $9.70(0.03)$ & $9.74(0.03)$ \\
\hline & 64 & $11.49(0.03)$ & $8.78(0.02)$ & $9.69(0.03)$ & $9.68(0.03)$ \\
\hline & 128 & $11.02(0.03)$ & $9.06(0.03)$ & $9.71(0.03)$ & $9.71(0.03)$ \\
\hline
\end{tabular}

Table 7

Down Out Call on three assets. Parameters analogous to Table 6 but for $D_{1}=D_{2}=$ $D_{3}=90$. Impact of the correlation $\rho$ on the accuracy of the numerical methods.

We define $\sigma_{B, t}$ its volatility at time $t$ by

$$
\frac{\mathrm{d} B_{t}}{B_{t}}=\cdots \mathrm{d} t+\sigma_{B, t} \mathrm{~d} W_{t}
$$

where $W$ is a Brownian motion. If $X_{i}$ has a dynamics of the form $\frac{\mathrm{d} X_{i, t}}{X_{i, t}}=$ $\cdots \mathrm{d} t+\sigma_{i, t}^{X} \mathrm{~d} W_{i, t}$ where the Brownian motions $\left(W_{i}\right)_{i}$ are correlated, then by 


\begin{tabular}{l|ll|ll|ll}
\hline & $\begin{array}{l}\text { Discrete } \\
\text { monitoring }\end{array}$ & $\begin{array}{l}\text { ABAST } \\
N\end{array}$ & $\begin{array}{l}\text { Discrete } \\
\text { monitoring } \\
D=85\end{array}$ & $\begin{array}{l}\text { ABAST } \\
D=95\end{array}$ & $\begin{array}{l}\text { Discrete } \\
\text { monitoring } \\
D=95\end{array}$ & $\begin{array}{l}\text { ABAST } \\
D=95\end{array}$ \\
\hline \hline 12 & 10.15 & 9.03 & 8.36 & 6.51 & 5.73 & 3.52 \\
\hline 24 & 9.90 & 9.06 & 7.88 & 6.49 & 4.95 & 3.20 \\
\hline 50 & 9.70 & 9.09 & 7.53 & 6.53 & 4.39 & 3.10 \\
\hline 100 & 9.51 & 9.07 & 7.22 & 6.50 & 4.00 & 3.06 \\
\hline 250 & 9.35 & 9.06 & 6.97 & 6.51 & 3.66 & 3.06 \\
\hline Table 8 & & & & &
\end{tabular}

Down Out Call on a Basket with 6 assets and two barriers. Accuracy of ABAST as $N$ increases. 1000000 simulations. For all the results, the statistical errors are smaller than 0.033 .

equating the quadratic variations of both sides of (34) we easily get

$$
\left[B_{t} \sigma_{B, t}\right]^{2}=\sum_{i, j} p_{i} p_{j} S_{i, t} S_{j, t} \sigma_{i, t}^{X} \sigma_{j, t}^{X} \rho_{i, j}
$$

This gives the value $\sigma_{B, t_{i}}$ which should be used to shift the barrier at time $t_{i}$. In Table 8 , we present numerical results for different values of the barriers. The aim of these tests is to check the accuracy of ABAST. Indeed, the usual discrete monitoring procedure still yields a slow convergence w.r.t. $N$ while for ABAST, it is much quicker. For a weekly monitoring (roughly $N=50$ ), this approach gives a bias smaller than the statistical error.

\subsection{BLAC Down Out option}

This example is aimed at illustrating the D2D approximation from Theorem 6. The Basket Lock Active Coupon (BLAC) Down Out option pays 1 Euro if at most one of 5 underlying assets has touched a lower barrier $L$ before the expiration. This is a bit more sophisticated than a simple barrier option. Usually, the assets are daily monitored to determine whether the barrier has been touched and if so, by how many assets. For contract expirations larger than 1 year, it may be computational demanding for the pricing and one may be interested in speeding it up by monitoring the assets only weekly or monthly and by adjusting the barrier $L$ (see Theorem 6 with $N_{1}=250$ and $N_{2}=52$ or $N_{2}=12$ ). Note that the barrier may be shifted differently for each asset because their volatilities may differ. In our experiments, the 5 assets $\left(X_{k}\right)_{k \leq 5}$ are modeled by correlated geometric Brownian motions, with 


\begin{tabular}{lllll}
\hline Barrier & True price & $\begin{array}{l}\text { ABAST } \\
N_{2}=52\end{array}$ & $\begin{array}{l}\text { ABAST } \\
N_{2}=26\end{array}$ & $\begin{array}{l}\text { ABAST } \\
N_{2}=12\end{array}$ \\
\hline \hline 70 & $0.519(0.001)$ & $0.519(0.001)$ & $0.517(0.001)$ & $0.512(0.001)$ \\
\hline 75 & $0.387(0.001)$ & $0.386(0.001)$ & $0.385(0.001)$ & $0.381(0.001)$ \\
\hline 80 & $0.2632(0.0009)$ & $0.2628(0.0009)$ & $0.2617(0.0009)$ & $0.2583(0.0009)$ \\
\hline 85 & $0.1578(0.0007)$ & $0.1578(0.0007)$ & $0.1559(0.0007)$ & $0.1551(0.0007)$ \\
\hline 90 & $0.0773(0.0005)$ & $0.0773(0.0005)$ & $0.0764(0.0005)$ & $0.0790(0.0005)$ \\
\hline
\end{tabular}

Table 9

BLAC Down Out option on 5 assets, with daily monitoring $N_{1}=250$. D2D ABAST approximation with weekly monitoring $N_{2}=52.1000000$ simulations. Values in parentheses indicate the half-width of the $95 \%$ confidence interval.

constant correlation $\rho=0.5$ and constant volatilities: $\sigma_{1}=0.3, \sigma_{2}=0.32$, $\sigma_{3}=0.35, \sigma_{4}=0.38, \sigma_{5}=0.4$. Their initial values are all equal to 100 and the interest rate equals 0 . The option expiration is $T=1$ year. We report in Table 9 firstly the prices computed by Monte Carlo simulations of the assets at $N_{1}$ dates, and secondly those with $N_{2}=52,26,12$ dates but with shifted barriers. The accuracy is very good and the computational time has been approximately divided by 5, 10 or 20 .

\section{References}

[1] Allaire, G., 2002. Shape optimization by the homogeneization method, 1st Edition. Springer Verlag, New-York.

[2] Baldi, P., 1995. Exact asymptotics for the probability of exit from a domain and applications to simulation. The Annals of Probability 23(4), 1644-1670.

[3] Bally, V., Talay, D., 1996. The law of the Euler scheme for stochastic differential equations: I. Convergence rate of the distribution function. Probab. Theory Related Fields 104-1, 43-60.

[4] Boyle, P., Lau, S., 1994. Bumping up against the barrier with the binomial method. Journal of Derivatives 1, 6-14.

[5] Boyle, P., Tian, Y., 1998. An explicit finite difference approach to the pricing of barrier options. Applied Mathematical Finance 5, 17-43.

[6] Broadie, M., Glasserman, P., Kou, S., 1997. A continuity correction for discrete barrier options. Mathematical Finance 7, 325-349.

[7] Broadie, M., Glasserman, P., Kou, S., 1999. Connecting discrete and continuous path-dependent options. Finance and Stochastics 3, 55-82. 
[8] Chang, J., Peres, Y., 1997. Ladder heights, Gaussian random walks and the Riemann zeta function. Ann. Probab. 25 (2), 787-802.

[9] Cheuk, T., Vorst, T., 1996. Complex barrier options. Journal of derivatives $4,8-22$.

[10] Costantini, C., Karoui, N. E., Gobet, E., 2006. Boundary sensitivities for diffusion processes in time dependent domains. Applied Mathematics and Optimization 54 (2), 159-187.

[11] Derman, E., Kani, I., Ergener, D., Bardhan, I., 1995. Enhanced numerical methods for options with barriers. Financial Analysts Journal Nov-Dec, 65-74.

[12] Gobet, E., 1999. Analysis of the zigzag convergence for barrier options with binomial trees. Tech. rep., Prépublication 536 du laboratoire PMA Paris 6 - http://www.proba.jussieu.fr/mathdoc/preprints/.

[13] Gobet, E., 2000. Euler schemes for the weak approximation of killed diffusion. Stochastic Processes and their Applications 87, 167-197.

[14] Gobet, E., 2001. Euler schemes and half-space approximation for the simulation of diffusions in a domain. ESAIM: Probability and Statistics 5, 261-297.

[15] Gobet, E., 2004. Revisiting the Greeks for European and American options. In: Akahori, J., Ogawa, S., Watanabe, S. (Eds.), Stochastic processes and applications to mathematical finance. World Scientific, pp. $53-71$.

[16] Gobet, E., Menozzi, S., 2004. Exact approximation rate of killed hypoelliptic diffusions using the discrete Euler scheme. Stochastic Processes and their Applications 112 (2), 201-223.

[17] Gobet, E., Menozzi, S., 2007. Discrete sampling of functionals of Itô processes. Séminaire de probabilités XL - Lecture Notes in Mathematics 1899 Springer Verlag, 355-374.

[18] Gobet, E., Menozzi, S., 2007. Stopped diffusion processes: Overshoots and boundary correction. Tech. rep., Preprint available on Arviv http://fr.arxiv.org/abs/0706.4042.

[19] Joe, H., 1997. Multivariate models and dependence concepts. Chapman \& Hall.

[20] Karatzas, I., Shreve, S., 1991. Brownian motion and stochastic calculus. Second Edition, Springer Verlag.

[21] Menozzi, S., 2006. Improved simulation for the killed Brownian Motion in a cone. SIAM Journal on Numerical Analysis 44 (6), 2610-2632.

[22] Merton, R., 1973. Theory of rational option pricing. Bell J. Econom. and Management Sci. 4, 141-183.

[23] Revuz, D., Yor, M., 1994. Continuous martingales and Brownian motion. 
2nd ed. Grundlehren der Mathematischen Wissenschaften. 293. Berlin: Springer.

[24] Ritchken, P., 1995. On pricing barrier options. Journal of derivatives 3, 19-28.

[25] Rogers, L., Stapleton, E., 1998. Fast accurate binomial pricing. Finance and Stochastics 2, 3-17.

[26] Rubinstein, M., Reiner, E., 1991. Breaking down the barriers. Risk 4(8), 28-35.

[27] Shevchenko, P., 2003. Addressing the bias in Monte Carlo pricing of multiasset options with multiple barriers through discrete sampling. Journal of Computational Finance 6 (3), 1-20.

[28] Siegmund, D., 1979. Corrected diffusion approximations in certain random walk problems. Adv. in Appl. Probab. 11 (4), 701-719.

\section{Appendix: numerical approximation of the expected overshoot of a Gaussian random walk}

The necessity of well approximating the expected overshoot $\bar{y}(u)=\mathbb{E}\left(s_{\tau^{u}}-u\right)$ for arbitrary levels $u$ is explained in Paragraph 2.4. It aims at reducing the simulation bias when the initial spot is close to the barriers.

From the numerical point of view, the computation of $\bar{y}(u)$ using a Monte Carlo method is not straightforward. The objective of this section is to provide a few related facts. On the one hand, it seems that one has only to simulate a Gaussian random walk $\left(s_{i}\right)_{1 \geq 0}$, until the hitting time $\tau^{u}$ of the level $u$ and then compute the overshoot $s_{\tau^{u}}-u$. On the other hand, although $\tau^{u}$ is finite with probability one, it has an infinite mean. This implies that our simulation procedure with $M$ independent paths will finish after some time, but it may take a huge computational time!!

To practically compute the expected overshoot, one should stop a simulation when it is too long, say when $\tau^{u}>t$ for a appropriate choice of a large $t$ to ensure a desired accuracy. For this, put

$$
\bar{y}(u, t):=\mathbb{E}\left(\left(s_{\tau^{u} \wedge t}-u\right)_{+}\right) .
$$

This is the quantity that we can compute by the simulation of the Gaussian random walk until time $t$ (or less). Since $s_{i}<u$ for $i<\tau^{u}$, one also has

$$
\bar{y}(u, t)=\mathbb{E}\left(\mathbf{1}_{\tau^{u} \leq t}\left(s_{\tau^{u}}-u\right)\right) .
$$


Thus, $\bar{y}(u, t)$ is increasing w.r.t. $t$ and by monotone convergence theorem, its limit as $t \rightarrow \infty$ is $\bar{y}(u)$. To estimate the approximation error, it is useful to derive upper bounds related to the overshoot.

Lemma 7 For any $p>0$ and for any $u>0$, one has

$$
\mathbb{E}\left(s_{\tau^{u}}-u\right)^{p} \leq \mathbb{E}\left|s_{1}\right|^{p} .
$$

PROOF. We embed the Gaussian random walk into a standard Brownian motion $W: s_{i}=W_{i}$. Put $\tau_{i}=\inf \left\{s>i: W_{s}=u\right\}$. Then, writing

$$
s_{\tau^{u}}-u=\sum_{i \geq 1} \mathbf{1}_{\tau^{u}>i-1}\left(s_{i}-u\right)_{+}=\sum_{i \geq 1} \mathbf{1}_{\tau^{u}>i-1} \mathbf{1}_{\tau_{i-1}<i}\left(W_{i}-W_{\tau_{i}}\right)_{+}
$$

and using the strong Markov property, the scaling invariance and the symmetry property of $W$, it follows that

$$
\begin{aligned}
\mathbb{E}\left(s_{\tau^{u}}-u\right)^{p} & =\sum_{i \geq 1} \mathbb{E}\left(\mathbf{1}_{\tau^{u}>i-1} \mathbf{1}_{\tau_{i-1}<i}\left(W_{i}-W_{\tau_{i}}\right)_{+}^{p}\right) \\
& \leq \sum_{i \geq 1} \mathbb{E}\left(\mathbf{1}_{\tau^{u}>i-1} \mathbf{1}_{\tau_{i-1}<i}\right) \mathbb{E}\left(\left[s_{1}\right]_{+}^{p}\right) \\
& =2 \mathbb{E}\left(\left[s_{1}\right]_{+}^{p}\right) \sum_{i \geq 1} \mathbb{E}\left(\mathbf{1}_{\tau^{u}>i-1} \mathbf{1}_{s_{i}>u}\right)=2 \mathbb{E}\left(\left[s_{1}\right]_{+}^{p}\right)=\mathbb{E}\left(\left|s_{1}\right|^{p}\right) .
\end{aligned}
$$

For $p=1$, by a direct computation $\mathbb{E}\left|s_{1}\right|$, we get the uniform upper bound

$$
\bar{y}(u) \leq 2 / \sqrt{2 \pi}=0.7979 .
$$

We recall from Chang and Peres [8] that $\bar{y}(0)=1 / \sqrt{2}=0.7071$ and $\bar{y}(\infty)=$ 0.5826 , which is not far from the upper bound.

To discuss the accuracy of $\bar{y}(u, t)$ for large $t$, we need to upper bound

$$
\begin{aligned}
& \mathbb{P}\left(\tau^{u}>t\right)=\mathbb{P}\left(\max _{i \leq t} W_{i}<u\right) \\
& \leq \mathbb{P}\left(\max _{s \leq t} W_{s}<u+\sqrt{3 \log t}\right)+\mathbb{P}\left(\max _{i \leq t} W_{i}<u ; \max _{s \leq t} W_{s} \geq u+\sqrt{3 \log t}\right) .
\end{aligned}
$$

The term $\sqrt{3 \log t}$ is chosen in order to approximately equate each contribution. The first one is equal to

$$
\mathbb{P}\left(\left|W_{t}\right|<u+\sqrt{3 \log t}\right) \leq \frac{2}{\sqrt{2 \pi t}}(u+\sqrt{3 \log t})
$$




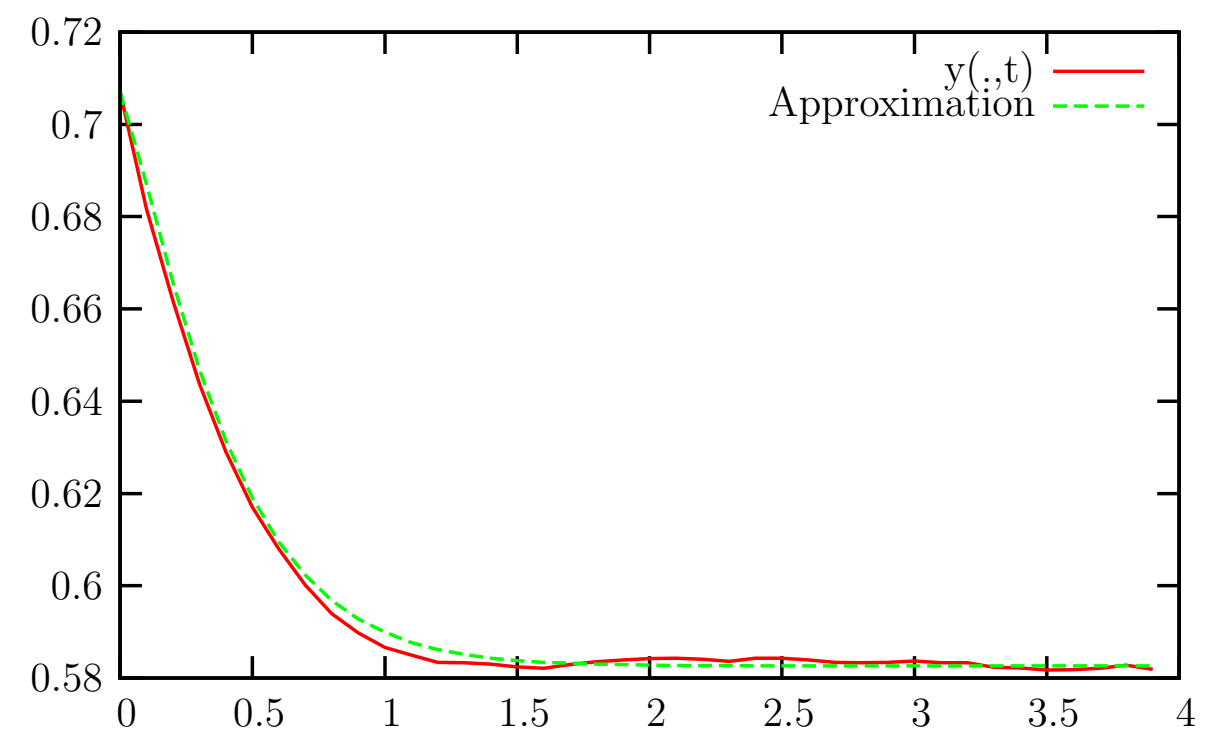

Figure 2. The numerical values of $\bar{y}(., t)$ and its approximation (38)

The second contribution is upper bounded by

$$
\begin{aligned}
\mathbb{P}\left(\exists i \leq t-1: \sup _{s \in[i, i+1]}\left(W_{s}-W_{i}\right)>\sqrt{3 \log t}\right) & \leq t \mathbb{P}\left(\left|W_{1}\right|>\sqrt{3 \log t}\right) \\
& \leq 2 t \exp \left(-(\sqrt{3 \log t})^{2} / 2\right)=\frac{2}{\sqrt{t}}
\end{aligned}
$$

By the Holder inequality, we deduce that for any $p \geq 1$ and $u \leq 4$

$$
\begin{aligned}
0 \leq \bar{y}(u)-\bar{y}(u, t) & =\mathbb{E}\left(\left(s_{\tau^{u}}-u\right) \mathbf{1}_{\tau^{u}>t}\right) \leq\left\|s_{\tau^{u}}-u\right\|_{2 p}\left(\mathbb{P}\left(\tau^{u}>t\right)\right)^{(2 p-1) /(2 p)} \\
& \leq\left(\frac{(2 p !)}{2^{p} p !}\right)^{1 /(2 p)}\left(\frac{2}{\sqrt{2 \pi t}}(4+\sqrt{2 \pi}+\sqrt{3 \log t})\right)^{(2 p-1) /(2 p)}
\end{aligned}
$$

For instance, an accuracy of 0.0025 is achieved for $t=3.15 \times 10^{8}$ (take $p=7$ in the upper bound). To tune the number of simulations $M$ in the evaluation of $\bar{y}(u, t)$, we note that the variance of $\left(s_{\tau^{u}}-u\right) \mathbf{1}_{\tau^{u} \leq t}$ is bounded by $\mathbb{E}\left(s_{1}^{2}\right)=1$ (owing to Lemma 7 ). Thus, $M=1.96^{2} / 0.0025^{2} \approx 615000$ yields a statistical error of order 0.0025 with probability $95 \%$, and thus an overall error of 0.005 (i.e. a posteriori less than $1 \%$ error).

The estimation of $\bar{y}(., t)$ that we obtain is plotted on Figure 2. A good approximation may be achieved using an exponential type function of the form

$$
\bar{y}(u) \approx \bar{y}(\infty)+(\bar{y}(0)-\bar{y}(\infty)) \exp \left(-2.7 u^{1.2}\right)
$$

with $\bar{y}(0)=0.7071$ and $\bar{y}(\infty)=0.5826$. It achieves an accuracy at least of $1 \%$. 